\title{
Energia cinética e pontos de equilíbrio de sistemas hamiltonianos
}

\author{
Renato Belinelo Bortolatto \\ DisSERTAÇÃO APRESENTADA \\ $\mathrm{AO}$ \\ Instituto De Matemática E EstatísticA \\ DA \\ Universidade de SÃo Paulo \\ PARA \\ OBTENÇÃO DO TÍTULO \\ $\mathrm{DE}$ \\ Mestre em CiênCIAS \\ Área de Concentração: Matemática Aplicada \\ Orientador: Prof. Dr. Fábio Armando Tal
}

Durante o desenvolvimento deste trabalho o autor recebeu auxílio financeiro do $\mathrm{CNPq}$

São Paulo, abril de 2008 


\section{Energia cinética e pontos de equilíbrio de sistemas hamiltonianos}

Este exemplar corresponde à redação final da dissertação devidamente corrigida e defendida por Renato Belinelo Bortolatto e aprovada pela Comissão Julgadora.

Banca Examinadora:

- Prof. Dr. Fábio Armando Tal (orientador) - IME-USP.

- Prof. Dr. Manuel Valentim de Pêra Garcia - IME-USP.

- Prof. Dr. Cláudio Aguinaldo Buzzi - UNESP. 


\section{Agradecimentos}

Para mim este trabalho é resultado de sete anos de dedicação exclusiva a um sonho. Apesar de ainda estar muito longe de quem eu gostaria de ser, eu vou (tentar) me permitir um momento de comemoração. Desta forma, tenho que começar agradecendo a professora Zara cuja paciência e carinho tem a cardinalidade da reta. Se a nós fosse dado o direito de escolher as companhias que temos nessa vida, ela seria minha primeira selecionada. Obrigado por tudo, professora.

Falando ainda dos professores, não posso esquecer de agradecer a professora Helena que me direcionou no caminho mais que preciso: eu ainda estou impressionado como sua indicação do orientador foi perfeita. Agradeço ao Fábio, que me recebeu de braços abertos como se tivesse reencontrado um velho amigo e me agraciou com o que é um de seus problemas favoritos. Agradeço que, mesmo com um talento digno de nota e responsabilidades pessoais e profissionais, está sempre disposto a ouvir minhas dúvidas e devaneios.

Aos amigos que apareceram nesses anos fica o desejo de que a vida permita que estejamos sempre juntos. Ao Ricardo e ao Eduardo, as mais novas adições nesta categoria, fica o agradecimento de ter sido recebido como um de vocês e as saudações acadêmicas pelas horas de estudo e papo-furado que passamos.

Agradeço também a toda minha família pelo apoio, principalmente meu pai, cujo incentivo é incondicional desde o primeiro dia. Eu não teria chegado até aqui sem você. Finalmente, agradeço a Aline por toda felicidade e paz que sua presença em minha vida me trouxe. É muito mais gostoso dividir os louros desse trabalho com todos vocês. 


\section{Resumo}

Estudaremos uma influência não trivial da energia cinética sobre pontos de equilíbrio de sistemas Hamiltonianos a partir da segunda parte do artigo de Garcia \& Tal The influence of the kinetic energy in equilibrium of Hamiltonian systems [5]. Nesse artigo demonstra-se, para um exemplo explícito de Hamiltonianos $C^{\omega}\left(\mathbb{R}^{4}\right)$ definidos por $H_{i}=T_{i}+\pi$ para $i \in\{1,2\}$, que as bacias de atração de $H_{1}$ e $H_{2}$ são subvariedades de $\mathbb{R}^{4}$ com dimensão distinta.

Discutiremos aqui de que forma esse resultado está relacionado com o estudo da estabilidade segundo Liapunov de pontos de equilíbrio de sistemas Hamiltonianos, em especial com a busca de uma inversão para o celebrado teorema de Dirichlet-Lagrange. Por fim apresentamos um novo teorema que estende o resultado acima para toda uma família de energias potenciais $\pi_{\alpha, \beta, k}$. A saber, mostramos que, se os parâmetros $\alpha, \beta, k$ satisfazem a um simples critério aritmético então as bacias de atração de $H_{i}=T_{i}+\pi_{\alpha, \beta, k}$ tem dimensões distintas para $i \in\{1,2\}$.

Palavras chave: Sistemas Hamiltonianos, energia cinética, bacia de atração. 


\begin{abstract}
We study a non trivial influence of the kinetic energy on equilibrium points of Hamiltonian systems following the second part of Garcia \& Tal article The influence of the kinetic energy in equilibrium of Hamiltonian systems [5]. In this article the authors show, for an explicit example of $C^{\omega}\left(\mathbb{R}^{4}\right)$ Hamiltonians defined by $H_{i}=T_{i}+\pi$ for $i \in\{1,2\}$, that the attraction basins of $H_{1}$ and $H_{2}$ have distinct dimensions as submanifolds of $\mathbb{R}^{4}$.

We'll discuss how this result is related to the study of the stability according to Liapunov of equilibrium points of Hamiltonian systems and especially how it is related to the inversion of the celebrated Lagrange-Dirichlet theorem. Finally we'll prove a new theorem which extends the result above for a whole family of potential energies $\pi_{\alpha, \beta, k}$. We show that, if the parameters $\alpha, \beta, k$ satisfy a simple arithmetical criteria then the attraction basins of $H_{i}=T_{i}+\pi_{\alpha, \beta, k}$ have different dimensions for $i \in\{1,2\}$.
\end{abstract}

Key-words: Hamiltonian systems, kinetic energy, attraction basin. 


\section{Sumário}

1 Introdução 1

2 Preliminares 3

$\begin{array}{lll}3 & \text { O teorema de Dirichlet-Lagrange } & 7\end{array}$

4 Alguns raciocínios preparatórios $\quad 13$

4.1 Duas funções importantes . . . . . . . . . . . . . . . . . 13

4.2 Encontrando soluções assintóticas à origem para $\ddot{x}=-k^{2}|x|^{\alpha} \quad 14$

4.3 Dois lemas de comparação . . . . . . . . . . . . . . 15

4.4 Duas desigualdades na família $(k t+c)^{-\alpha} \ldots \ldots \ldots$

5 Otimizando um expoente $\quad 19$

6 Um critério explícito 31

$\begin{array}{lll}7 & \text { Conclusões } & 43\end{array}$

8 Apêndice $\quad 45$

8.1 Apêndice ao capítulo $5 \ldots \ldots \ldots \ldots \ldots$

8.2 Apêndice ao capítulo $6 \ldots \ldots \ldots$. . . . . . . . 46

Referências Bibliográficas $\quad 49$ 


\section{Capítulo 1}

\section{Introdução}

Não é difícil encontrar argumentos para afirmar que uma parte considerável do que hoje consideramos matemática foi fortemente influenciada pelo estudo da mecânica analítica clássica no século XVIII. Surpreendentemente, a formulação matemática moderna para a mecânica, amada por uns e odiada por tantos, ainda inspira desenvolvimentos no campo da matemática abstrata e é fonte de inúmeros problemas de enunciado simples que resistiram as tentativas dos séculos.

Um exemplo disso é o problema da estabilidade (no sentido de Liapunov) de pontos de equilíbrio de sistemas hamiltonianos, o assunto ao qual esta dissertação está fortemente relacionada. Apesar de um dos enunciados principais da teoria datar de 1788, uma demonstração válida só foi obtida em 1846. Esse enunciado, conhecido hoje como teorema de Dirichlet-Lagrange, propõe condições sobre a energia potencial de um sistema conservativo para garantir a estabilidade de um ponto de equilíbrio. Novos resultados que supondo estabilidade, além de hipóteses pouco restritivas, forneçam condições sobre a energia potencial ainda são muito desejáveis.

O leitor atento pôde perceber que foi evitada a palavra "recíproca" no último parágrafo. De fato, P. Painlevé [13] em 1904, forneceu um contraexemplo para tal hipótese. Contudo, o caso em que a energia potencial é analítica foi estudado por Palamodov e admite tal recíproca, pelo menos onde a energia potencial não tem mínimo.

Essa linha de pesquisa quase exclui a possibilidade de que a energia cinética desempenhe papel na caracterização de estabilidade para sistemas mecânicos. Isso começou a mudar nos últimos dez anos. Um resultado de Bertotti e Bolotin [3] em 2000 mostra a existência de uma energia potencial $C^{\infty}$ cujo equilíbrio é estável para uma energia cinética $T_{1}$ e instável para $T_{2}$, ambas $C^{\infty}$. Já em 2005, Garcia e Tal [5] apresentaram um exemplo explícito, mais simples e inédito, da influência da energia cinética na estabilidade do equilíbrio para hamiltonianos $C^{k}$. Mais do que isso o caso analítico é estudado e outro exemplo é fornecido para mostrar que a energia cinética 
pode modificar a dimensão da bacia de atração do equilíbrio. O estudo deste último resultado é o objetivo principal desta dissertação.

A dissertação foi dividida em sete capítulos, contando com essa introdução, além de um apêndice. O capítulo 2 fala dos conceitos básicos de equações diferenciais, estabilidade e mecânica e foi pensado também como forma de fixar as notações. O capítulo 3 trata do teorema de DirichletLagrange e sua recíproca, localizando nosso estudo no contexto histórico e fornecendo as principais referências. O capítulo 4 explicita alguns raciocínios que serão usados constantemente na demonstração principal. O capítulo 5 e o capítulo 6 apresentam generalizações do artigo original: o primeiro de forma a encontrar o menor expoente possível para um dos termos da energia potencial e o segundo proporciona um critério algébrico simples para determinar se o fenômeno estudado ocorre para um potencial da forma fixada (o leitor familiarizado com a área e interessado apenas no resultado principal pode pular diretamente do capítulo 4 para o capítulo 6). Por fim, o sétimo capítulo discute as conclusões obtidas e finaliza este trabalho. Na sequência está um breve apêndice dedicado a alguns cálculos que foram apenas indicados nos capítulos 5 e 6 .

Ao leitor esclareço que um grande esforço foi feito para que sua leitura seja agradável, sem pecar pelo excesso ou pela falta, mas peço desculpas antecipadamente pelas partes obscuras e erros que inevitavelmente aparecerão. Boa leitura. 


\section{Capítulo 2}

\section{Preliminares}

Este capítulo tem a finalidade de familiarizar o leitor com os conceitos e notações que serão utilizados no decorrer desta dissertação. Não há o intuito de apresentar um resumo completo nem estritamente formal dos grandes temas de equações diferenciais e mecânica analítica clássica que serão abordados. Apesar disso, estes poucos parágrafos devem ser o suficiente para que os leitores com algum conhecimento da área sintam-se convidados a continuar sua tarefa.

Antes, algumas palavras sobre a notação adotada. O símbolo 0 será usado indistintamente para representar o elemento neutro em sua versão escalar e em sua versão vetorial. O contexto ficará encarregado de indicar qual significado deve ser tomado.

Definição 2.1. Seja $\Omega=\operatorname{int}(\Omega) \subseteq \mathbb{R}^{n}$. Dada uma função $f: \mathbb{R} \times \Omega \rightarrow \mathbb{R}^{n} o$ problema de Cauchy consiste em determinar, para um dado par $\left(t_{0}, x_{0}\right) \in$ $\mathbb{R} \times \Omega$, uma solução $x:] t_{-}, t_{+}\left[\rightarrow \mathbb{R}^{n}\right.$ (com $\left.t_{0} \in\right] t_{-}, t_{+}[$) para a equação diferencial ordinária (EDO)

$$
\frac{d x(t)}{d t}=f(t, x(t)), \quad x\left(t_{0}\right)=x_{0}
$$

onde a condição $x\left(t_{0}\right)=x_{0}$ é conhecida como condição inicial.

Também representaremos $\frac{d x(t)}{d t}$ simplesmente por $\dot{x}$, que é a notação mais tradicional em mecânica. Uma EDO é dita autônoma se $f(t, x)$ não depende da variável $t$, ou seja, se pudermos escrever $f(t, x)=\tilde{f}(x)$.

Se supormos, por exemplo, que $f \in C^{1}\left(\mathbb{R} \times \Omega\right.$ ) (onde $C^{k}(\mathbb{R} \times \Omega$ ) é o conjunto das funções com todas k-ésimas derivadas parciais contínuas em $\mathbb{R} \times$ $\Omega$ ) podemos garantir a existência e unicidade de uma solução para qualquer problema de Cauchy, ao menos em uma vizinhança suficientemente pequena de $t_{0}$. Além disso, essa solução depende continuamente da condição inicial $\left(t_{0}, x_{0}\right)$, que será omitida como variável na expressão da solução $x\left(t ; t_{0}, x_{0}\right)$ de (2.1) sempre que conveniente, a exemplo do que já foi feito nos parágrafos anteriores. 
No decorrer desse texto poderíamos pedir apenas que o problema de Cauchy satisfaça algum teorema de existência, unicidade e dependência contínua com relação às condições iniciais, sem nos preocuparmos com a hipótese específica que garanta essas propriedades. Contudo, a hipótese $f \in C^{1}(\mathbb{R} \times \Omega)$ aparece naturalmente nesse estudo e será escolhida por sua suficiência e simplicidade, além do fato de se encaixar muito bem no contexto da mecânica clássica. Estaremos também sempre considerando a solução maximal, isto é, a solução de (2.1) definida no maior intervalo em torno de $t_{0}$ (ou seja, a solução $x: I \rightarrow \mathbb{R}^{n}$ tal que para qualquer outra solução $y: J \rightarrow \mathbb{R}^{n}$ temos $\left.t_{0} \in J \subseteq I\right)$.

Definição 2.2. Dizemos que $x_{0} \in \mathbb{R}^{n}$ é um ponto de equilíbrio de (2.1) se a função satisfazendo $x(t)=x_{0}$ para todo $t \in \mathbb{R}$ é uma solução de (2.1).

Note que sob hipóteses que garantam existência e unicidade essa definição é equivalente a pedir que $f(t, x)=0$, para todo $t \in \mathbb{R}$, no problema de Cauchy dado por (2.1). Sempre que possível consideraremos que $t_{0}=0$ e que $x_{0}=0$ via mudanças de coordenadas adequadas.

Definição 2.3. Uma solução $x(t)$ é estável segundo Liapunov em $t=t_{0}$ se está definida para todo $t \geq t_{0}$ e para todo $\varepsilon>0$ existe $\delta>0$ tal que se $\left\|x\left(t_{0}\right)-\bar{x}\left(t_{0}\right)\right\|<\delta$ então $\|x(t)-\bar{x}(t)\|<\varepsilon$ para todo $t \geq t_{0}$, onde $\|$.$\| é uma$ norma qualquer para o $\mathbb{R}^{n}$ e $\bar{x}(t)$ é uma solução, em geral diferente de $x(t)$, para o problema de Cauchy dado por (2.1) e a condição inicial $\bar{x}\left(t_{0}\right)$.

Uma solução é instável se ela não for estável, ou seja, se não está definida para todo $t \geq t_{0}$ ou se, dado $\delta>0$, existe alguma solução $\bar{x}(t)$ de forma que $\left\|x\left(t_{0}\right)-\bar{x}\left(t_{0}\right)\right\|<\delta$ mas $\|x(t)-\bar{x}(t)\| \geq \varepsilon$ para algum $t \geq t_{0} .{ }^{1}$

Definição 2.4. Se uma solução é estável segundo Liapunov em $t=t_{0} e$ todas as soluçôes $\bar{x}(t)$ de (2.1) com condição inicial em alguma vizinhança de $x\left(t_{0}\right)$ se aproximam de $x(t)$ quando $t \rightarrow \infty$ dizemos que a solução $x(t)$ é assintóticamente estável.

Diremos que uma solução $x(t)$ do problema de Cauchy (2.1) é assintótica a um ponto de equilibrio $x_{0} \in \mathbb{R}^{n}$ se $\lim _{t / \infty} x(t)=x_{0}$.

Definição 2.5. A bacia de atração de um ponto de equilíbrio $x_{0}$ é o conjunto das condições iniciais cuja solução do problema de Cauchy associado é assintótica a $x_{0}$. Ela será denotada por $B A\left(x_{0}\right)=\left\{(t, y) \in \mathbb{R}^{n+1}\right.$ : $\left.\lim _{t / \infty} x(t)=\left\{x_{0}\right\}\right\}$.

As definições acima servem para estudar a estabilidade segundo Liapunov para qualquer tipo de solução, mas como o caso que queremos estudar

\footnotetext{
${ }^{1}$ Em especial, a hipótese $f \in C^{1}$ pedida anteriormente implica que $x$ está definida para todo $t \geq t_{0}$. Não iremos nos aprofundar nesse raciocínio, que é parte de um curso básico de equações diferenciais.
} 
trata especificamente de estabilidade de soluções passando por pontos de equilíbrio, podemos dizer que um ponto é estável segundo Liapunov ao invés de dizer que uma solução é estável segundo Liapunov.

Como vimos, determinar a estabilidade de uma solução depende do conhecimento a priori de todas as soluções em uma vizinhança de $x(t)$. O (segundo) método de Liapunov é um resultado bem conhecido que permite sanar essa deficiência por meio de funções auxiliares. Uma versão desse método para o caso autônomo é a seguinte

Teorema 2.6 (Segundo método de Liapunov). Seja $x_{0}$ um ponto de equilíbrio de (2.1). Se existe uma função diferenciável $V: \Omega \rightarrow \mathbb{R}$ (onde $\Omega$ é uma vizinhança aberta de $x_{0} \in \mathbb{R}^{n}$ ) tal que

1. $V\left(x_{0}\right)=0$

2. $V(x)>0$, para todo $x$ diferente de $x_{0}$

3. $\frac{d V}{d t}(x(t)) \leq 0$, para toda solução $x(t)$ de (2.1)

Então o ponto $x_{0}$ é estável segundo Liapunov. Além disso, se fortalecermos a terceira hipótese para

3'. $\frac{d V}{d t}(x(t))<0$ para toda solução $x(t)$ de (2.1)

então o ponto $x_{0}$ será assintóticamente estável.

Apesar de $V$ ser calculada sobre as trajetórias, é possível verificar se $V(x)$ é positiva para todo $x \in \Omega$ e podemos usar a regra da cadeia para verificar se $V$ é não-crescente sobre as trajetórias, ou seja, podemos aplicar o teorema acima mesmo sem o conhecimento explícito de todas as soluções de (2.1). Contudo, ainda resta o problema de achar uma função $V$, o que geralmente é muito difícil.

Ainda tratando de sistemas autônomos temos, como alternativa, um método "mais simples"que consiste somente em linearizar a função $f(x)$ em um ponto de equilíbrio $x_{0}$ e determinar a parte real dos autovalores de $D f_{x_{0}}$ : um autovalor com parte real positiva garante a instabilidade enquanto todos autovalores com parte real negativa garantem a estabilidade assintótica. Apesar de imediata, essa abordagem só pode garantir estabilidade assintótica, o que não ocorre em sistemas com integrais primeiras (ou seja, sistemas que possuem funções não triviais que sejam constantes sobre as trajetórias). No estudo de sistemas mecânicos conservativos, que são o foco dessa dissertação, a energia total do sistema é uma integral primeira e portanto o (segundo) método de Liapunov será, de certa forma, nossa ferramenta principal.

Há mais de uma maneira de estudar sistemas mecânicos. Nesse trabalho estaremos usando a abordagem hamiltoniana, apesar da abordagem lagrangiana ser também uma ferramenta poderosa no estudo da estabilidade 
de pontos de equilíbrio de sistemas conservativos. Um campo hamiltoniano pode ser definido por uma função real $H: \mathbb{R}^{2 n} \rightarrow \mathbb{R}$ de classe $C^{2}$ que satisfaz, se escrevemos $(q, p) \in \mathbb{R}^{n} \times \mathbb{R}^{n}$, às equações de Hamilton

$$
\left\{\begin{array}{c}
\dot{q}=\frac{\partial H}{\partial p} \\
\dot{p}=-\frac{\partial H}{\partial q}
\end{array}\right.
$$

contudo, no caso dos sistemas mecânicos naturais (com os quais estaremos trabalhando) consideramos especificamente a função "energia total" $H(q, p)=T(q, p)+\pi(q)$, onde a "energia cinética" é dada pela expressão $T(q, p)=\frac{1}{2} p^{T} B(q) p$ para uma matriz simétrica definida positiva $B(q) \in C^{2}$ e a "energia potencial" $\pi(q) \in C^{2}$.

Por meio de uma mudança de coordenadas, supomos também que a energia potencial $\pi$ satisfaz $\pi(0)=0$ e $\frac{\partial \pi(q)}{\partial q}(0)=0$, ou seja, que o ponto de equilíbrio de (2.2) que desejamos estudar está na origem.

A teoria dos sistemas hamiltonianos é muito rica e interessante, mas não abordaremos neste texto seus aspectos intuitivos nem seus principais resultados. Há uma grande variedade de livros dedicados a esse assunto (por exemplo [9] e [2]) detalhando essa teoria por vias elementares, intermediárias ou até mesmo filosóficas. No próximo capítulo iremos colocar essas definições em prática estudando os principais resultados sobre a estabilidade de pontos de equilíbrio para sistemas mecânicos conservativos, mantendo o foco em nosso estudo proposto inicialmente. 


\section{Capítulo 3}

\section{O teorema de Dirichlet-Lagrange}

Este capítulo é dedicado ao teorema de Dirichlet-Lagrange e as tentativas de sua inversão. A referência básica pra este capítulo é [15] e alguns resultados mais recentes são citados a seguir.

Com a definição abaixo estaremos prontos para apresentar uma demonstração do teorema principal desta seção

Definição 3.1. Dizemos que um sistema hamiltoniano dado por $H(q, p)=$ $T(q, p)+\pi(q)$ satisfaz as hipóteses usuais se estiver definido em um aberto $\Omega$ que contém o vetor nulo $(0,0), T(q, p)=\frac{1}{2} p^{T} B(q) p$ para uma matriz simétrica definida positiva $B(q) \in C^{2}(\Omega)$ e $\pi(q) \in C^{2}(\Omega)$ satisfaz $\pi(0)=0$, $\frac{\partial \pi(q)}{\partial q}(0)=0$.

Teorema 3.2 (Dirichlet-Lagrange). Seja $H(q, p)=T(q, p)+\pi(q)$ satisfazendo as hipóteses usuais. Se supormos adicionalmente que $\pi(q)>0$ para $q \in \Omega \operatorname{com} q \neq 0$ então a origem $(0,0)$ é estável segundo Liapunov para o sistema (2.2)

$$
\left\{\begin{array}{c}
\dot{q}=\frac{\partial H}{\partial p} \\
\dot{p}=-\frac{\partial H}{\partial q}
\end{array}\right.
$$

Demonstração. A demonstração seguirá diretamente do segundo método de Liapunov apresentado no capítulo anterior se usarmos como função auxiliar $V=H$. De fato, note que

1. $H(0,0)=0$

2. Como $B(q)$ é uma função contínua, positiva definida em $q=0$ temos que $H(q, p) \geq H(q, 0)=\pi(q)>0$. 
3. $H(q, p)$ é constante sobre as trajetórias de (2.2). De fato, temos que

$$
\frac{d H(q(t), p(t))}{d t}=\dot{q}(t) \frac{\partial H(q(t), p(t))}{\partial q}+\dot{p}(t) \frac{\partial H(q(t), p(t))}{\partial p}=0
$$

O teorema de Dirichlet-Lagrange é informalmente enunciado como " um ponto de mínimo estrito é estável". É importante notar a falta de uma informação indispensável ao seu entendimento: Ponto de mínimo estrito para qual função? Essa maneira informal de traduzir o conteúdo do teorema de Dirichlet-Lagrange reflete o ponto de vista dominante (ou melhor, que costumava a ser o dominante) da pesquisa na área, isto é, que a descrição da estabilidade em sistemas mecânicos poderia ser completamente entendida apenas pela energia potencial.

O problema de inverter o teorema de Dirichlet-Lagrange, ou melhor, achar condições necessárias para a estabilidade segundo Liapunov de sistemas mecânicos conservativos pode ser justificado de duas importantes maneiras:

- É muito conveniente nas aplicações tecnológicas sabermos a priori se um experimento ou uma máquina funciona de forma estável, ou seja, se pequenas variações nas condições iniciais não apresentarão empecilho ao seu funcionamento da forma planejada.

- O interesse matemático natural de inverter teoremas e caracterizar propriedades.

Para encontrar pistas sobre a inversão do teorema de Dirichlet-Lagrange podemos primeiro enfraquecer as hipóteses e tentar uma demonstração por um método energético direto, ao invés de recorrer ao segundo método de Liapunov. Temos o

Teorema 3.3. Suponha além das hipóteses usuais que para todo $\rho>0 \mathrm{com}$ $\overline{B(0, \rho)} \subseteq$ domínio $(\pi)$ exista um aberto $U \subseteq \mathbb{R}^{n}$ tal que $0 \in U \subseteq B(0, \rho)$ e que para todo $q \in \partial U$ temos que $\pi(q)>0$. Então a origem $(0,0)$ é um ponto de equilíbrio estável segundo Liapunov.

Da forma como está apresentado, esse teorema pode não parecer muito mais abrangente que o teorema de Dirichlet-Lagrange. Contudo, ele pode ser usado para fornecer um exemplo de ponto de equilíbrio estável segundo Liapunov que não é um ponto de mínimo estrito. De fato, o 
Exemplo 3.4 (Winter, 1941). ${ }^{1}$ Seja

$$
\pi(q)=\left\{\begin{array}{c}
e^{-\frac{1}{q^{2}}} \cos \left(\frac{1}{q}\right), \text { se } q \neq 0 \\
0, q=0
\end{array}\right.
$$

Note que $q=0$ não é ponto de mínimo estrito, mas $(0,0)$ é estável, o que pode ser visto aplicando o teorema anterior.

Com esse exemplo, perdemos as esperanças de que o teorema de Dirichlet -Lagrange, como apresentado no início desse capítulo, possua uma recíproca perfeita. A versão mais abrangente que apresentamos acima também não admite recíproca exata (a não ser quando possuímos apenas um grau de liberdade). Para ver isso temos o seguinte exemplo:

Exemplo 3.5 (Laloy, 1975). Seja $T\left(q_{1}, q_{2}, p_{1}, p_{2}\right)=\frac{1}{2}\left(p_{1}^{2}+p_{2}^{2}\right) e$

$$
\pi\left(q_{1}, q_{2}\right)=\left\{\begin{array}{c}
e^{-\frac{1}{q_{1}^{2}}} \cos \left(\frac{1}{q_{1}}\right)-e^{-\frac{1}{q_{2}^{2}}}\left(\cos \left(\frac{1}{q_{2}}\right)+q_{2}^{2}\right), \text { se } q_{1}, q_{2} \neq 0 \\
-e^{-\frac{1}{q_{2}^{2}}}\left(\cos \left(\frac{1}{q_{2}}\right)+q_{2}^{2}\right), \text { se } q_{1}=0 \\
e^{-\frac{1}{q_{1}^{2}}} \cos \left(\frac{1}{q_{1}}\right), \text { se } q_{2}=0 \\
0, \text { se } q_{1}=q_{2}=0
\end{array}\right.
$$

Veja que sob a reta $q_{1}=q_{2}$ temos que $\pi\left(q_{1}, q_{2}\right)=-e^{-\frac{1}{q_{2}^{2}}} q_{2}^{2}<0$ se $\left(q_{1}, q_{2}\right) \neq(0,0)$, e portanto não existe nenhum aberto em torno da origem para o qual todo ponto da fronteira satisfaça $\pi\left(q_{1}, q_{2}\right)>0$. Contudo, as equações de hamilton

$$
\left\{\begin{array}{c}
\dot{q}_{1}=p_{1} \quad \dot{q}_{2}=p_{2} \\
\dot{p_{1}}=-\frac{\partial \pi\left(q_{1}, q_{2}\right)}{\partial q_{1}} \quad \dot{p_{2}}=-\frac{\partial \pi\left(q_{1}, q_{2}\right)}{\partial q_{2}}
\end{array}\right.
$$

podem ser separadas em dois sistemas independentes aos quais podemos aplicar o teorema anterior de formas similares.

No exemplo acima vimos pela primeira vez uma hipótese não usual sobre a energia cinética $T\left(q_{1}, q_{2}, p_{1}, p_{2}\right)$ (na verdade, a hipótese é muito específica, já que pede uma forma específica para essa função). Não conhecemos resultados sobre a estabilidade da origem desse sistema com outras possíveis energias cinéticas. Contudo, somente esse exemplo não constitui evidência suficiente para suspeitarmos que exista alguma influência da energia cinética na estabilidade do equilíbrio. De fato, outros três resultados indicam o caminho oposto:

\footnotetext{
${ }^{1}$ Este exemplo pode ser creditado a Painlevé por sua nota [13] publicada em 1904, pois o exemplo fornecido naquela ocasião é perfeitamente similar, mas apenas $C^{5}$. Neste capítulo optamos por distinguir esses exemplos e destacar a versão $C^{\infty}$, atribuída a Winter (veja $[15]$ ).
} 
Teorema 3.6 (Hagedorn [7], 1971). Além das hipóteses usuais suponha adicionalmente que $\pi(q)$ tem um ponto de máximo estrito na origem. Então $(0,0)$ é instável segundo Liapunov.

Teorema 3.7 (Taliaferro [16], 1980). Além das hipóteses usuais suponha adicionalmente que $\pi(q)$ tem um ponto de máximo (não necessariamente estrito) na origem. Então $(0,0)$ é instável segundo Liapunov.

Estes resultados, elusivamente simples, foram obtidos usando a versão lagrangiana da mecânica clássica, ou seja, usando o princípio da ação crítica e contornando as dificuldades técnicas com muita engenhosidade.

Teorema 3.8 (Palamodov [14], 1995). Além das hipóteses usuais suponha adicionalmente que $H(q, p)$ é analítica em uma vizinhança da origem. Se $\pi(0)$ não for um ponto de mínimo então $(0,0)$ é instável segundo Liapunov.

O teorema de Palamodov apresenta uma inversão quase completa do teorema de Dirichlet-Lagrange para o caso em que $H$ é analítica (o problema de determinar condições brandas sobre o potencial para garantir a estabilidade de um ponto de mínimo não estrito ainda está aberto para $n$ graus de liberdade, mesmo nesse caso). Esse resultado é, portanto, mais um forte indício ${ }^{2}$ de que a energia cinética não desempenha qualquer papel na estabilidade do equilíbrio.

Ainda poderíamos citar outros resultados nessa direção, por exemplo N. G. Chetaev (1952), forneceu um resultado interessante que decide a instabilidade do equilíbrio sempre que $\left(\frac{\partial \pi(q)}{\partial q} \mid q\right)<0$ para todo $q$ no conjunto em que $\pi(q)<0$. Mais recentemente temos os resultados de Moauro \& Negrini [11] (1980) e [10] (1991) que determinam a instabilidade quando $\pi(q)$ é um polinômio homogêneo mais um resto e por fim o resultado de Garcia \& Tal [6] (2003), na mesma direção, mas usando o conceito de k-decidibilidade como proposto por Barone Netto [12] (1984). Notamos, mais uma vez, que todos esses resultados não exigem hipóteses adicionais sobre a energia cinética.

Assim, só podemos imaginar a surpresa com que, em 2000, o artigo de Bertotti \& Bolotin [3] foi recebido por parte do meio acadêmico, já que o artigo mostrou que existe uma energia potencial $\pi(q) \in C^{\infty}$ e duas energias cinéticas distintas $T_{1}, T_{2} \in C^{\infty}$ tais que o equilíbrio da origem é estável para $H_{1}(q, p)=T_{1}(q, p)+\pi(q)$ e instável para $H_{2}(q, p)=T_{2}(q, p)+\pi(q)$. Em 2005 o artigo de Garcia \& Tal [5] no qual se baseia essa dissertação foi publicado exibindo um exemplo simples do mesmo resultado que Bertotti \& Bolotin [3], só que para um caso um pouco diferente em que as energias cinéticas são $C^{\omega}$ (ao invés de apenas $C^{\infty}$ ) e a energia potencial envolvida é $C^{k}$, mas não $C^{\infty}$. Na segunda parte desse mesmo artigo eles mostraram que existe influência não trivial da energia cinética na dimensão da bacia

\footnotetext{
${ }^{2}$ Convém notar que a demonstração desse resultado teve sua porção final apenas indicada.
} 
de atração da origem, mesmo no caso em que o hamiltoniano é analítico. Nos capítulos a seguir analisamos esse resultado, corrigindo uma parte do raciocínio (seguindo a sugestão dada pelos próprios autores) e regulando os expoentes dos polinômios envolvidos na função potencial que foi originalmente exibida. Contudo, vamos primeiramente para alguns raciocínios preparatórios. 


\section{Capítulo 4}

\section{Alguns raciocínios preparatórios}

Dedicamos esse capítulo a alguns raciocínios bem simples, mas que serão fundamentais nos dois capítulos seguintes. Eles serão usados mais de uma vez, assim optamos por justificá-los aqui e destacamos seu uso no decorrer do texto.

\subsection{Duas funções importantes}

A função módulo de um número real, é claro, não é diferenciável na origem. Mesmo assim podemos escrever a fórmula algébrica de sua derivada como $\frac{|x|}{x}$ fora do ponto $x=0$ (de fato ela vale -1 se $x<0$ e 1 se $x>0$ ).

Desejamos verificar que a função $|x|^{a} \operatorname{com} a>1$ é diferenciável para todo $x \in \mathbb{R}$. Para isso note que fora de $x=0$ sabemos que ela é diferenciável (e, portanto, contínua) pois se $x>0$ ela vale $a x^{a-1}$ e se $x<0$ vale $-a(-x)^{a-1}$. Para garantirmos a diferenciabilidade na origem basta observar que os limites laterais coincidem (pois $\lim _{x \backslash 0} a x^{a-1}=\lim _{x \succ 0}-a(-x)^{a-1}$ ) e são iguais a $f^{\prime}(0)=\lim _{x \rightarrow 0} \frac{f(x)}{x}= \pm \lim _{x \rightarrow 0} x^{a-1}=0$ (pois $\alpha-1>0$ ). Com o que discutimos acima e a ajuda da regra da cadeia veja que

$$
\frac{d|x|^{a}}{d x}=\left\{\begin{array}{c}
a \frac{|x|^{a}}{x}, \text { se } x \neq 0 \\
0, \text { se } x=0
\end{array}\right.
$$

Estaremos estudando de perto o comportamento das funções $f(x)=$ $x|x|^{a}$ e $g(x)=-k|x|^{b}$. Note que é preciso algum cuidado para lidar com a função $f(x)$, pois $f(0)=0$ (e por isso não é necessário excluir o ponto $x=0$ do seu domínio de definição quando $a>1$ ). Veja também que a função $x|x|^{a}$ vale $x^{a+1}$ quando $x>0 \mathrm{e}-|x|^{a+1}$ quando $x<0$. Como $a+1>2$ a função $x|x|^{a}$ é $C^{2}(\mathbb{R})$. Para garantir que $-k|x|^{b}$ seja função $C^{2}(\mathbb{R})$ com mínimo em zero pediremos que $b \geq 2$ e $k>0$. 
Usaremos também que $f(x)=x|x|^{a}$ tem um ponto de sela na origem. Conforme discutimos nessa seção é fácil ver que sua derivada é $(a+1)|x|^{a} \mathrm{e}$ que portanto a função $x|x|^{a}$ tem de fato um ponto crítico na origem. Como a função $x|x|^{a}$ é negativa para $x<0$ e positiva para $x>0$ concluímos que ela tem um ponto de sela na origem. Em resumo

Lema 4.1.1. Se $a>1$ e $b \geq 2$ as funções $f(x)=x|x|^{a}$ e $g(x)=-k|x|^{b}$ são $C^{2}(\mathbb{R})$ e suas derivadas são $f^{\prime}(x)=(a+1)|x|^{a}$ e $g^{\prime}(x)=-k b \frac{|x|^{b}}{x}$. Além disso $f(x)$ possui um ponto de sela na origem $e$, se $k>0$, a função $g(x)$ possui um ponto de máximo na origem.

\subsection{Encontrando soluções assintóticas à origem para}

$$
\ddot{x}=-k^{2}|x|^{\alpha}
$$

Desejamos encontrar todas as soluções assintóticas à origem de $\ddot{x}=-k^{2}|x|^{\alpha}$ para $k \neq 0$ e $\alpha>1$ (note que assim estamos nas hipóteses do lema acima). Para isso definimos, como $a>1$,

$$
V(x, \dot{x})=\frac{\dot{x}^{2}}{2}+\frac{k^{2}}{\alpha+1} x|x|^{\alpha}
$$

Uma trajetória assintótica à origem satisfaz $\lim _{t \rightarrow+\infty} V(x, \dot{x})=0$. Se observarmos que $\dot{V}=\dot{x} \ddot{x}+\dot{x} k^{2}|x|^{\alpha}=0$ então $V$ será constante e igual a zero sobre estas trajetórias, ou seja,

$$
\frac{\dot{x}^{2}}{2}=\frac{-k^{2}}{\alpha+1} x|x|^{\alpha} \Longrightarrow \dot{x}= \pm \sqrt{\frac{2 k^{2}}{\alpha+1}} \sqrt{|x|^{\alpha+1}}
$$

pois $x \leq 0$ (o que pode ser visto notando que $\frac{\dot{x}^{2}}{2} \geq 0$ ). Note que, se $x\left(t_{0}\right)=0$ então $\dot{x}\left(t_{0}\right)=0$ e obtemos apenas a solução constante $x_{t}=0$, para todo $t \in \mathbb{R}$. Desta forma, note também que $x<0$ implica que $\dot{x}>0$ para uma trajetória assintótica à origem, pois caso contrário $\lim _{t \rightarrow \infty} x(t) \neq 0$. Assim

$$
\dot{x}=\sqrt{\frac{2 k^{2}}{\alpha+1}} \sqrt{|x|^{\alpha+1}} \Longleftrightarrow \frac{\dot{x}}{|x|^{\frac{\alpha+1}{2}}}=\sqrt{\frac{2 k^{2}}{\alpha+1}}
$$

Cuja solução é

$$
x=\left(\left(\frac{-\alpha+1}{2}\right)\left(\sqrt{\frac{2 k^{2}}{\alpha+1}}\right) t+C_{0}\right)^{\frac{2}{-\alpha+1}}
$$

o que pode ser verificado por derivação, lembrando que temos $x<0$, e onde $C_{0}^{\frac{2}{-\alpha+1}}=x(0)$.

Se usarmos na definição de $V$ a subtração dos termos ao invés da soma podemos gerar soluções assintóticas satisfazendo $x>0$ (e, portanto, $\dot{x}<0$ ). 
Lidaremos também com as equações da forma $\ddot{x}=k^{2} \frac{|x|^{\beta}}{x}$. Este caso pode ser facilmente reduzido ao anterior se supormos que $x$ é positivo ou negativo assim obtemos um resultado análogo sem esforço adicional.

Note também que as soluções obtidas acima são assintóticas à origem (pois o expoente é negativo).

\subsection{Dois lemas de comparação}

O lema abaixo é um conhecido teorema de equações diferenciais de primeira ordem

Lema 4.3.1. Sejam $u(t, x), v(t, x) \in C^{1}(I \times U)$ (onde $U, I \subseteq \mathbb{R}$ são intervalos abertos com $x_{0} \in U$ e $\left[t_{0}, t_{f}[\subset I)\right.$. Se $u(t, x)<v(t, x)$ para todo $(t, x) \in I \times U$ e se $\phi(t), \psi(t)$ são as (únicas) soluções, definidas para todo $t \in\left[t_{0}, t_{f}\left[\right.\right.$, das equações diferenciais $\dot{x_{1}}=u(t, x)$ e $\dot{x_{2}}=v(t, x)$, com condições iniciais $\phi\left(t_{0}\right)=\psi\left(t_{0}\right)=x_{0}$ então temos que $\phi(t)<\psi(t)$ para todo $t \in] t_{0}, t_{f}[$.

Demonstração. Note que $t_{0} \in A=\left\{\tau \in\left[t_{0}, t_{f}[: \phi(t)=\psi(t)\}\right.\right.$. Quero mostrar que $A=\left\{t_{0}\right\}$.

Em $t_{0}$ temos que $\left.\frac{d \phi}{d t}\right|_{t=t_{0}}<\left.\frac{d \psi}{d t}\right|_{t=t_{0}}$, portanto em um intervalo suficientemente pequeno $] t_{0}, t_{0}+\varepsilon_{1}[$ temos $\phi(t)<\psi(t)$ (pois $\phi(t)-\psi(t)$ é decrescente neste intervalo e vale zero em $\left.t_{0}\right)$. Portanto nenhum elemento de $] t_{0}, t_{0}+\varepsilon_{1}[$ está em $A$.

É fácil ver de modo análogo que os pontos de $A$ são isolados, portanto, como $A$ é fechado, podemos supor por um momento que existe um elemento $T \neq t_{0}$ em $A$ tal que $T \leq s$, para todo $s \in A \backslash\left\{t_{0}\right\}$. Então existe um $\varepsilon_{2}>0$ tal que em $] T-\varepsilon_{2}, T[$ temos $\phi(t)>\psi(t)$ (pois $\phi(t)-\psi(t)$ é crescente neste intervalo e vale zero em $T$ ), o que é uma contradição, pois o teorema do valor intermediário garante que existiria $s<T$ tal que $s \in A \backslash\left\{t_{0}\right\}$.

Os sistemas mecânicos naturais são determinados por equações diferenciais de segunda ordem, portanto o lema acima não pode ser aplicado diretamente. Estamos interessados em um lema de comparação que trate desta situação, ao menos no caso das trajetórias assintóticas à origem para o sistema da seção anterior. O lema a seguir é uma resposta para este problema, e é uma das idéias centrais na demonstração de nossos teoremas principais.

Lema 4.3.2. Sejam $k \neq 0$ e $\alpha>1$ dados e seja $\phi(t)$ uma solução assintótica à origem satifazendo $\ddot{\phi}<-k^{2}|\phi|^{\alpha}$ e $\psi(t)$ uma solução assintótica à origem para o sistema $\ddot{x}=-k^{2}|x|^{\alpha}$. Se $\phi\left(t_{0}\right)=\psi\left(t_{0}\right)=x_{0}$ então $\phi(t)>\psi(t)$, para todo $t>t_{0}$ no domínio comum das soluções.

Demonstração. Associamos a $\ddot{x}<-k^{2}|x|^{\alpha}($ para $k \neq 0)$ a função auxiliar $V(x, \dot{x})$ definida para $\alpha>1$ 


$$
V(x, \dot{x})=\frac{\dot{x}^{2}}{2}+\frac{k^{2}}{\alpha+1} x|x|^{\alpha}
$$

Observamos inicialmente que $\dot{V}=\dot{x} \ddot{x}+\dot{x} k^{2}|x|^{\alpha}<0$ se $\dot{x}>0$ e $\dot{V} \geq 0$ caso contrário.

Como $\ddot{x}<0$ para todo $x \neq 0$ (pois supomos $k \neq 0$ ), sabemos que $\dot{x}$ é decrescente fora da origem. Assim, para que $(x(t), \dot{x}(t))$ seja uma trajetória assintótica à origem, temos obrigatoriamente que ter $\dot{x}>0$. Concluímos que $V$ é decrescente e como para uma trajetória assintótica temos $\lim _{t \rightarrow+\infty} V(x, \dot{x})=0$ então $V>0$, isto é,

$$
\frac{\dot{x}^{2}}{2}>\frac{-k^{2}}{\alpha+1} x|x|^{\alpha}
$$

Além disso como $\dot{x}>0$ e estamos considerando trajetórias assintóticas à origem podemos conluir que $x<0, \operatorname{logo}$

$$
\dot{x}>\sqrt{\frac{2 k^{2}}{\alpha+1}} \sqrt{|x|^{\alpha+1}}
$$

Usamos o lema 4.3.1 para concluir que

$$
x>\left(\left(\frac{-\alpha+1}{2}\right)\left(\sqrt{\frac{2 k^{2}}{\alpha+1}}\right) t+C_{0}\right)^{\frac{2}{-\alpha+1}}
$$

para todo $t>t_{0}$, onde $C_{0}^{\frac{2}{-\alpha+1}}=x_{0}$. Note que, como $\alpha>1$, o termo da direita é negativo, o que concorda com o fato que $x<0$.

Portanto as assintóticas à origem para a inequação $\ddot{x}<-k^{2}|x|^{\alpha}$ estão dominadas pelas soluções assintóticas à origem para o sistema $\ddot{x}=-k^{2}|x|^{\alpha}$, para $k \neq 0$ e $\alpha>1$ dados, conforme determinamos na seção anterior.

\subsection{Duas desigualdades na família $(k t+c)^{-\alpha}$}

Vamos utilizar nos próximos dois capítulos as desigualdades na família $h(k, c, \alpha)=(k t+c)^{-\alpha}$ para $\alpha>0$ que são dadas pelos dois lemas abaixo.

Lema 4.4.1. Se $0<k_{1}<k_{2}$ e $0<a<b$ então, para algum $t$ suficientemente grande temos que $h\left(k_{2}, c_{2}, b\right)<h\left(k_{1}, c_{1}, a\right)$. Nos referimos a esta desigualdade como "majoramos pelo maior expoente", devido ao sinal negativo na definição de $h(k, c, \alpha)$.

Demonstração. Como $0<k_{1}<k_{2}$ temos, para $t>\left|\frac{c_{1}-c_{2}}{k_{1}-k_{2}}\right|$, que

$$
\left(k_{1} t+c_{1}\right)-\left(k_{2} t+c_{2}\right)=\left(k_{1}-k_{2}\right) t+\left(c_{1}-c_{2}\right)<0
$$


Consequentemente para $t>0$ suficientemente grande valerá que $k_{1} t+$ $c_{1}<k_{2} t+c_{2}$. Usando uma idéia análoga veja, usando a hipótese $0<a<b$, que

$$
\left(k_{1} t+c_{1}\right)^{-a}-\left(k_{2} t+c_{2}\right)^{-b}=\frac{\left(k_{2} t+c_{2}\right)^{b}-\left(k_{1} t+c_{1}\right)^{a}}{\left(k_{1} t+c_{1}\right)^{a}\left(k_{2} t+c_{2}\right)^{b}}
$$

Como $k_{1} t+c_{1}<k_{2} t+c_{2}$ para $t>\left|\frac{c_{1}-c_{2}}{k_{1}-k_{2}}\right|$ podemos escolher $t>$ $\max \left\{\left|\frac{c_{1}-1}{k_{1}}\right|,\left|\frac{c_{1}-c_{2}}{k_{1}-k_{2}}\right|\right\}$ para que $1<k_{1} t+c_{1}<k_{2} t+c_{2}$. Por sua vez $t^{a}$ é crescente quando $a>0$. Portanto $\left(k_{1} t+c_{1}\right)^{a}<\left(k_{2} t+c_{2}\right)^{a}$ e como $0<a<b$ e os termos entre parênteses são maiores que 1 temos que $\left(k_{2} t+c_{2}\right)^{a}<\left(k_{2} t+c_{2}\right)^{b}$. Portanto $\left(k_{1} t+c_{1}\right)^{a}<\left(k_{2} t+c_{2}\right)^{b}$ e podemos tomar $t$ suficientemente grande de forma que

$$
\left(k_{1} t+c_{1}\right)^{-a}-\left(k_{2} t+c_{2}\right)^{-b}>0 \Longrightarrow\left(k_{1} t+c_{1}\right)^{-a}>\left(k_{2} t+c_{2}\right)^{-b}
$$

Concluímos que, para $t$ suficientemente grande temos que $\left(k_{1} t+c_{1}\right)^{-a}>$ $\left(k_{2} t+c_{2}\right)^{-b}$, ou seja, $h\left(k_{2}, c_{2}, b\right)<h\left(k_{1}, c_{1}, a\right)$.

Lema 4.4.2. Caso $0<k_{1}<k_{2}$ e $0<a=b$ temos para algum $t$ suficientemente grande que $h\left(k_{2}, c_{2}, a\right)<h\left(k_{1}, c_{1}, a\right)$. Nos referimos a esta desigualdade como "quando os expoentes são iguais, majoramos pelo menor termo que multiplica $t "$.

Demonstração. Essa demonstração segue idêntica a anterior, até o pen-último parágrafo do lema anterior, onde basta observar que $\left(k_{1} t+c_{1}\right)^{a}<$ $\left(k_{2} t+c_{2}\right)^{a}$ para concluir que, para $t$ suficientemente grande, $\left(k_{1} t+c_{1}\right)^{-a}>$ $\left(k_{2} t+c_{2}\right)^{-a}$, ou seja $h\left(k_{2}, c_{2}, a\right)<h\left(k_{1}, c_{1}, a\right)$. 


\section{Capítulo 5}

\section{Otimizando um expoente}

Neste capítulo começamos o estudo da influência da energia cinética na dimensão da bacia de atração do equilíbrio que temos na origem dos eixos coordenados de uma família de sistemas hamiltonianos. Analisamos o problema seguindo de perto o artigo de Garcia \& Tal [5], com a exceção de que o termo que fazia o papel de uma "direção" com máximo na origem era o termo fixado $-q_{2}^{10}$ que no nosso caso será um termo mais geral apresentando máximo na origem da forma $-k\left|q_{2}\right|^{\beta}$ com as hipóteses adicionais $k>0$, para garantir que 0 seja um ponto de máximo, e $\beta \geq 2$ para garantir que os hamiltonianos envolvidos sejam $C^{2}\left(\mathbb{R}^{4}\right)$. Contudo, em nossa abordagem pediremos $\beta>2$ o que não constitui um grande problema, já que o teorema principal dessa seção exige uma condição mais forte. Além disso, apresentamos uma correção no texto original, sugerida pelos próprios autores, que consiste de uma versão particular do lema 5.4 e parte da demonstração do teorema.

Esse capítulo é um passo intermediário na direção de transformar o exemplo apresentado originalmente em uma classe de exemplos de potenciais que são influenciados pelas energias cinéticas $T_{1}, T_{2}$ conforme apresentadas a seguir. Neste primeiro momento nos concentraremos em encontrar o menor $\beta$ possível, tentando diminuir o expoente em $-q_{2}^{10}$ apresentado no artigo original.

Intuitivamente, a constante $k>0$ faz o papel de controlar a "força" da "direção"com máximo na origem. Ela aparece para validar algumas desigualdades (lemas 4.4.1 e 4.4.2) e garantir o melhor resultado possível dentro do raciocínio proposto. No capítulo seguinte refinaremos esse resultado olhando para o termo $q_{1}^{3}$, atingindo assim nosso resultado principal.

Seja, para $\beta>2$ e $k>0$ dados,

$$
\begin{gathered}
\pi: \Omega=\left\{\left(q_{1}, q_{2}\right) \in \mathbb{R}^{2}: q_{1}>-1\right\} \rightarrow \mathbb{R} \\
\pi\left(q_{1}, q_{2}\right)=\frac{q_{1}^{3}-k\left|q_{2}\right|^{\beta}}{2}
\end{gathered}
$$


e considere

$$
\begin{gathered}
T_{1}, T_{2}: \Omega \times \mathbb{R}^{2} \rightarrow \mathbb{R}^{+} \\
T_{1}=\frac{p_{1}{ }^{2}+p_{2}{ }^{2}}{2}, \quad T_{2}=\frac{\left(1+q_{1}\right)\left(p_{1}{ }^{2}+p_{2}{ }^{2}\right)}{2}
\end{gathered}
$$

Se definirmos $H_{1}=T_{1}+\pi$, obtemos que

$$
H_{1}\left(q_{1}, q_{2}, p_{1}, p_{2}\right)=\frac{p_{1}^{2}+p_{2}^{2}}{2}+\frac{q_{1}^{3}-k\left|q_{2}\right|^{\beta}}{2}
$$

cujas equações hamiltonianas são

$$
\left\{\begin{array}{cc}
\dot{q_{1}}=p_{1} & \dot{q}_{2}=p_{2} \\
\dot{p_{1}}=-\frac{3}{2} q_{1}^{2} & \dot{p_{2}}=k \frac{\beta}{2} \frac{\left|q_{2}\right|^{\beta}}{q_{2}}
\end{array}\right.
$$

O lema 4.1.1 garante que este sistema está bem definido e é $C^{1}\left(\mathbb{R}^{4}\right)$. Em particular, para condições iniciais adequadas, as equações $\ddot{q}_{1}=-\frac{3}{2} q_{1}{ }^{2} \mathrm{e}$ $\ddot{q_{2}}=k \frac{\beta}{2} \frac{\left|q_{2}\right|^{\beta}}{q_{2}}$ tem como possíveis soluções

$$
q_{1}(t)=\frac{-4}{\left(t+c_{0}\right)^{2}}, \quad q_{2}(t)=\left(\frac{4}{k(\beta-2)^{2}}\right)^{\frac{1}{\beta-2}}\left|t+c_{1}\right|^{\frac{-2}{\beta-2}}
$$

para $t>\max \left\{-c_{0},-c_{1}\right\}$, ambas assintóticas à origem. Caso estivéssemos lidando com o caso $\beta=2$ teríamos que $q_{2}=\exp \left(-\sqrt{\frac{k \beta}{2}} t+c_{3}\right)$ é uma solução assintótica à origem, mas não da mesma forma que as soluções descritas acima. Por isso esse caso não está contemplado no que segue, apesar de boa parte das afirmações abaixo ainda serem válidas.

A análise da bacia de atração do sistema (5.1) será simplificada se definirmos as funções

$H_{1,1}\left(q_{1}(t), p_{1}(t)\right)=\frac{p_{1}^{2}(t)}{2}+\frac{q_{1}^{3}(t)}{2}, \quad H_{1,2}\left(q_{2}(t), p_{2}(t)\right)=\frac{p_{2}^{2}(t)}{2}+\frac{-k\left|q_{2}(t)\right|^{\beta}}{2}$

Lema 5.1. A bacia de atração da origem para o sistema (5.1) é o conjunto

$$
\begin{gathered}
B A_{1}=\left\{\left(q_{1}, q_{2}, p_{1}, p_{2}\right) \in \Omega \times \mathbb{R}^{2}: H_{1,1}\left(q_{1}, p_{1}\right)=0=H_{1,2}\left(q_{2}, p_{2}\right),\right. \\
\left.p_{1} q_{1} \leq 0 \text { e } p_{2} q_{2} \leq 0\right\}
\end{gathered}
$$

Demonstração. Seja $\phi(t)=\left(q_{1}, q_{2}, p_{1}, p_{2}\right)(t)$ uma solução de (5.1) assintótica à origem e $I$ seu intervalo maximal de definição. Temos que

$$
H_{1}\left(q_{1}(t), q_{2}(t), p_{1}(t), p_{2}(t)\right)=0, \quad \forall t \in I
$$

pois $H_{1}$ é contínua, constante sobre as soluções e $H_{1}(0,0,0,0)=0$. Portanto, como temos $H_{1}=H_{1,1}+H_{1,2}=0$ para todo $t$, vale que $H_{1,1}\left(q_{1}, p_{1}\right)=$ 
- $H_{1,2}\left(q_{2}, p_{2}\right)$ Um cálculo simples mostra que uma solução de (5.1) satisfaz $\dot{H}_{1,1}=\dot{H}_{1,2}=0$. Além disso $\lim _{t / \infty} H_{1,1}\left(q_{1}(t), p_{1}(t)\right)=0$ pois $\left(q_{1}, q_{2}, p_{1}, p_{2}\right)(t)$ é uma solução de (5.1) assintótica à origem. Note então que, como acima, essas observações implicam que $H_{1,1}\left(q_{1}, p_{1}\right)=0=H_{1,2}\left(q_{2}, p_{2}\right)$

Além disso se uma solução é assintótica à origem, como $\left(p_{2} q_{2}\right)=k \frac{\beta}{2}\left|q_{2}\right|^{\beta}+$ $p_{2}^{2} \geq 0$, a função $p_{2}(t) q_{2}(t)$ é crescente para todo $t$ e deve tender a zero. Logo $p_{2} q_{2} \leq 0$. Analogamente note que $H_{1,1}=0$ implica que $q_{1} \leq 0$ que por sua vez garante que $\left(p_{1} q_{1}\right)=-\frac{3}{2} q_{1}^{3}+p_{1}^{2} \geq 0$. Portanto $p_{1} q_{1} \leq 0$.

Por outro lado considere uma solução passando por $\left(q_{1}, q_{2}, p_{1}, p_{2}\right) \in \Omega \times$ $\mathbb{R}^{2}$ e tal que $H_{1,1}\left(q_{1}, p_{1}\right)=H_{1,2}\left(q_{2}, p_{2}\right)=0, p_{1} q_{1} \leq 0, p_{2} q_{2} \leq 0$. Quero mostrar que ela é assintótica à origem. Como $H_{1}=H_{1,1}+H_{1,2}$ temos que $H_{1}=0$. Note também que $H_{1,1}\left(q_{1}, p_{1}\right)=0$ mostra que $q_{1}=0$ se e só se $p_{1}=0$ e de forma análoga conclua que $p_{2}=0$ se e só se $q_{2}=0$.

Temos então alguns casos para estudar:

1. O caso $(0,0,0,0)$ é trivial, pois a trajetória $(0,0,0,0)$ é a única solução assintótica à origem.

2. Para os casos $\left(q_{1}(0), 0, p_{1}(0), 0\right)$ onde $p_{1}(0) q_{1}(0)<0$ e $\left(0, q_{2}(0), 0, p_{2}(0)\right)$ e $p_{2}(0) q_{2}(0)<0$ note que os planos $(X, 0, Y, 0)$ e $(0, X, 0, Y)$ são invariantes pelas soluções de (5.1). Podemos utilizar então as soluções encontradas na seção 4.2 para construir uma solução assintótica à origem da forma $\left(q_{1}(t), 0, p_{1}(t), 0\right)$ ou $\left(0, q_{2}(t), 0, p_{2}(t)\right)$ conforme o caso estudado.

3. Finalmente, para o caso em que $q_{1}(0) p_{1}(0)<0$ e $q_{2}(0) p_{2}(0)<0$, construímos uma solução assintótica à origem a partir das soluções para $H_{1,1}$ e $H_{1,2}=0$ encontradas na seção 4.2.

Assim, como cada conjunto está contido no outro, obtivemos sua igualdade.

Façamos agora a analise de $H_{2}=T_{2}+\pi$

$$
H_{2}\left(q_{1}, q_{2}, p_{1}, p_{2}\right)=\frac{\left(1+q_{1}\right)\left(p_{1}^{2}+p_{2}^{2}\right)}{2}+\frac{q_{1}^{3}-k\left|q_{2}\right|^{\beta}}{2}
$$

cujas equações hamiltonianas são,

$$
\left\{\begin{array}{cc}
\dot{q}_{1}=\left(1+q_{1}\right) p_{1} & \dot{q_{2}}=\left(1+q_{1}\right) p_{2} \\
\dot{p_{1}}=-\frac{3}{2} q_{1}{ }^{2}-\frac{\left(p_{1}{ }^{2}+p_{2}{ }^{2}\right)}{2} & \dot{p_{2}}=k \frac{\beta}{2} \frac{\left|q_{2}\right|^{\beta}}{q_{2}}
\end{array}\right.
$$

Novamente, o lema 4.1.1 garantirá que este sistema está bem definido e é $C^{1}\left(\mathbb{R}^{4}\right)$. Isso, em particular, quer dizer que se tomarmos condições iniciais 
$\left(q_{1}, q_{2}, p_{1}, p_{2}\right)(0)$ tais que $p_{2}(0)=q_{2}(0)=0$ teremos $p_{2}(t)=q_{2}(t)=0$ para todo $t$, o que pode ser verificado por substituição e usando a unicidade das soluções. Portanto, as equações para $\dot{p}_{1}$ e $\dot{q}_{1}$ sobre uma solução satisfazendo essa condição inicial são

$$
\dot{p_{1}}=-\frac{3}{2} q_{1}^{2}-\frac{1}{2} p_{1}^{2}, \quad \dot{q_{1}}=\left(1+q_{1}\right) p_{1}
$$

e, portanto, $\left(q_{1}, p_{1}\right)$ satisfazem as equações hamiltonianas para $\tilde{H}_{2}\left(q_{1}, p_{1}\right)=$ $\left(1+q_{1}\right) \frac{p_{1}^{2}}{2}+\frac{q_{1}^{3}}{2}$. Como a energia potencial (unidimensional) associada a esse sistema tem ponto de sela na origem note que as equações hamiltonianas de $\tilde{H}_{2}$ possuem uma trajetória assintótica à origem.

No que segue, o objetivo é mostrar que a condição $p_{2}(t)=q_{2}(t)=0$, válida para todo $t$ onde uma solução estiver definida, é necessária para que uma solução de (5.3) seja assintótica à origem.

Vamos começar com poucas observações sobre a natureza das soluções assintóticas à origem para esse sistema em particular. Seja então $\phi(t)=$ $\left(q_{1}(t), q_{2}(t), p_{1}(t), p_{2}(t)\right)$ solução de (5.3) tal que $\lim _{t \rightarrow+\infty}\|\phi(t)\|=0$. Estamos estudando apenas as trajetórias assintóticas, então podemos considerar o tempo $t_{0}$ tal que, para todo $t>t_{0},\left|q_{1}(t)\right|<\frac{1}{2}$ (assumimos $t_{0}=0$ sem perda de generalidade). Veja que

$$
\dot{p_{1}}=-\frac{3}{2} q_{1}^{2}-\frac{\left(p_{1}^{2}+p_{2}^{2}\right)}{2} \leq 0
$$

i.e., $p_{1}$ é não-crescente. Então, para que a solução seja assintótica, só podemos ter que $p_{1}(t) \geq 0$, para todo $t \in \mathbb{R}$, já que $\lim _{t \rightarrow+\infty}\|\phi(t)\|=0$. Assim

$$
\dot{q}_{1}=\left(1+q_{1}\right) p_{1} \geq \frac{1}{2} p_{1} \geq 0
$$

i.e., $q_{1}$ é não-decrescente e portanto necessariamente valerá $q_{1}(t) \leq 0$ para todo $t \in \mathbb{R}^{+}$, como argumentamos acima.

Note, usando (5.3), que $\dot{p}_{1}(0)=0$ se e somente se $q_{1}(0)=p_{1}(0)=$ $p_{2}(0)=0$. Sobre uma trajetória assintótica $q_{1}(t)$ é não-decrescente e tende zero, portanto nesse caso teremos $q_{1}(t)=0$ para todo $t>0$ e como $p_{1}(t)$ é não-crescente e tende zero temos também que $p_{1}(t)=0$ para todo $t>0$. Desta forma $-\frac{p_{2}^{2}(t)}{2}=0$ para todo $t>0$ e assim temos que $\dot{q}_{2}=\left(1+q_{1}\right) p_{2}=$ 0 , ou seja, para que essa trajetória seja assintótica à origem, precisamos ter $q_{2}(t)=0$ para todo $t>0$. Desta forma mostramos que se $\dot{p_{1}}(0)=0$ então a solução $\phi(s)$ será constante e igual ao ponto $(0,0,0,0)$, para todo $s>0$.

No interesse de considerar apenas as soluções assintóticas à origem com $p_{2}(s), q_{2}(s) \neq 0$ para algum $s>0$, podemos considerar, daqui em diante $p_{1}(t)>0$ para todo $t \in \mathbb{R}^{+}$e, consequentemente, $q_{1}(t)<0$ para todo $t \in \mathbb{R}^{+}$.

Desta forma, para uma trajetória assintótica e para todo $t>0$, temos que $0>q_{1}(t)>-\frac{1}{2}$. Como $k>0$ valerá que 


$$
\left(q_{2} p_{2}\right)=\left(1+q_{1}\right) p_{2}^{2}+k \frac{\beta}{2}\left|q_{2}\right|^{\beta} \geq \frac{1}{2} p_{2}^{2}+k \frac{\beta}{2}\left|q_{2}\right|^{\beta} \geq 0
$$

Concluímos portanto que $q_{2}(t) p_{2}(t) \leq 0$, pois $\lim _{t \rightarrow+\infty}\|\phi(t)\|=0$. Mais ainda, se olharmos para as equações

$$
\left\{\begin{array}{c}
\dot{q_{2}}=\left(1+q_{1}\right) p_{2} \\
\dot{p_{2}}=k \frac{\beta}{2} \frac{\left|q_{2}\right|^{\beta}}{q_{2}}
\end{array}\right.
$$

podemos ver que $p_{2}(\tau)=0$ se e somente se $q_{2}(\tau)=0$, para algum $\tau \in \mathbb{R}$. De fato, se $p_{2}(\tau)=0$ e $q_{2}(\tau)>0$ temos que $\dot{p_{2}}(\tau)>0$, i.e., $p_{2}(\tau)$ é localmente crescente. Assim a continuidade das equações deixa afirmar que teríamos um intervalo $] \tau, \tau_{1}$ [ em que $p_{2}>0$ e $q_{2}>0$, o que contradiz $q_{2}(t) p_{2}(t) \leq 0$. O caso $p_{2}(\tau)=0$ e $q_{2}(\tau)<0$ é análogo, assim como os dois restantes. Se tomarmos condições iniciais $\left(q_{1}(0), q_{2}(0), p_{1}(0), p_{2}(0)\right)$ tais que $p_{2}(0)=q_{2}(0)=0$ teremos $p_{2}(t)=q_{2}(t)=0$ usando a unicidade de soluções. Desta forma podemos supor sem perda de generalidade que $q_{2}(t) p_{2}(t)<0$ para todo $t>0$, pois não estamos interessados nas soluções que satisfazem $p_{2}(t)=q_{2}(t)=0$ para todo $t \in \mathbb{R}$.

Passamos agora para três resultados intermediários que utilizam as informações obtidas nos últimos parágrafos e funções auxiliares para comparar o comportamento entre as funções coordenadas de soluções que sejam assintóticas à origem. Note que esses resultados e suas respectivas demonstrações estão fortemente relacionadas com o segundo lema de comparação que apresentamos no capítulo 4 (lema 4.3.2).

Lema 5.2. Se $\phi(t)$ é solução de (5.3) tal que $\lim _{t \rightarrow+\infty}\|\phi(t)\|=0$ então para todo $t>0$ temos $p_{1}(t)>\left(-q_{1}(t)\right)^{\frac{3}{2}}$

Demonstração. Defina $V\left(q_{1}(t), p_{1}(t)\right)=\frac{q_{1}^{3}(t)+p_{1}^{2}(t)}{2}$. Temos

$$
\dot{V}\left(q_{1}, p_{1}\right)=\frac{3}{2} q_{1}^{2}\left(1+q_{1}\right) p_{1}+p_{1}\left(-\frac{3}{2} q_{1}^{2}-\frac{p_{1}^{2}+p_{2}^{2}}{2}\right)=\frac{p_{1}}{2}\left(3 q_{1}^{3}-p_{1}^{2}-p_{2}^{2}\right)<0
$$

Como $\lim _{t \rightarrow+\infty} V\left(q_{1}(t), p_{1}(t)\right)=0$ e $V$ é decrescente concluímos que $V\left(q_{1}(t), p_{1}(t)\right)<0$, ou seja, $\frac{q_{1}^{3}+p_{1}^{2}}{2}>0$ pois $p_{1}>0$ e $q_{1}<0$ para todo $t>0$

Lema 5.3. Se $\phi(t)$ é solução de (5.3) tal que $\lim _{t \rightarrow+\infty}\|\phi(t)\|=0$ e $p_{2}(\tau) q_{2}(\tau) \neq$ 0 para algum $\tau>0$ então para todo $t>0$ temos $\left|p_{2}(t)\right|>\sqrt{k}\left|q_{2}(t)\right|^{\frac{\beta}{2}}$ 
Demonstração. Defina $U\left(q_{2}(t), p_{2}(t)\right)=\frac{-k\left|q_{2}(t)\right|^{\beta}+p_{2}^{2}(t)}{2}$. Como

$$
\dot{U}\left(q_{2}, p_{2}\right)=-k \frac{\beta}{2} \frac{\left|q_{2}\right|^{\beta}}{q_{2}}\left(1+q_{1}\right) p_{2}+p_{2}\left(k \frac{\beta}{2} \frac{\left|q_{2}\right|^{\beta}}{q_{2}}\right)=-k \frac{\beta}{2} \frac{\left|q_{2}\right|^{\beta}}{q_{2}} q_{1} p_{2}<0
$$

A ultima desigualdade segue da seguinte forma: sabemos que $q_{1}(t)<0$ para todo $t>0$ e que $p_{2}(\tau) q_{2}(\tau)<0$. Desta forma $p_{2}(t) q_{2}(t) \neq 0$ para todo $t$ (como já argumentamos anteriormente). Assim $q_{2}(t) p_{2}(t)<0$ e podemos dividir por $q_{2}^{2}(t)$ para concluir que $\frac{p_{2}(t)}{q_{2}(t)}<0$. Desta forma as hipóteses implicam que $U\left(q_{2}(t), p_{2}(t)\right)>0, \operatorname{logo}\left|p_{2}(t)\right|>\sqrt{k}\left|q_{2}(t)\right|^{\frac{\beta}{2}}$

Lema 5.4. Se $\phi$ é solução de (5.3) tal que $\lim _{t \rightarrow+\infty}\|\phi(t)\|=0$ e $p_{2}(\tau) q_{2}(\tau) \neq$ 0 para algum $\tau>0$ então para todo $t>0$ temos $\left|p_{2}(t)\right|<\sqrt{2 k}\left|q_{2}(t)\right|^{\frac{\beta}{2}}$

Demonstração. Defina $U_{2}\left(q_{1}(t), q_{2}(t), p_{2}(t)\right)=\left(1+q_{1}(t)\right) \frac{p_{2}^{2}(t)}{2}-k \frac{\left|q_{2}(t)\right|^{\beta}}{2}$ e veja que

$\dot{U}_{2}\left(q_{1}, q_{2}, p_{2}\right)=\frac{p_{2}^{2}}{2} \dot{q_{1}}+\left(1+q_{1}\right) p_{2} \dot{p_{2}}-k \frac{\beta}{2} \frac{\left|q_{2}\right|^{\beta}}{q_{2}} \dot{q_{2}}=\frac{p_{2}^{2}}{2} \dot{q_{1}}=\frac{p_{2}^{2}}{2}\left(1+q_{1}\right) p_{1}>0$

pois $\left|q_{1}(t)\right|<\frac{1}{2}$ e $p_{1}(t)>0$, para todo $t>0$. Como vimos $p_{2}(\tau)=0$ se e somente se $q_{2}(\tau)=0, \operatorname{logo} U_{2}\left(q_{1}(t), q_{2}(t), p_{2}(t)\right) \neq 0$, pois caso contrário $p_{2}(t) q_{2}(t)=0$. Além disso, por hipótese $\lim _{t \rightarrow+\infty}\|\phi(t)\|=0$, então temos que $U_{2}\left(q_{1}(t), q_{2}(t), p_{2}(t)\right)<0$ para todo $t>0 \mathrm{e}$, como $-\frac{1}{2}<q_{1}(t)<0$ para todo $t>0$, temos

$$
\left(1+q_{1}\right) \frac{p_{2}^{2}}{2}<k \frac{\left|q_{2}\right|^{\beta}}{2} \Longrightarrow p_{2}^{2}<2 k\left|q_{2}\right|^{\beta}
$$

Procedemos com o intuito de mostrar que a condição $p_{2}(t)=q_{2}(t)=0$ para todo $t$ é necessária para que uma solução de (5.3) seja assintótica à origem. Até agora só precisamos que $k>0$ e $\beta>2$ como condições de regularidade (lema 4.1.1). Infelizmente as contas abaixo exigem um pouco mais do que isso. Por razões de objetividade incluímos a hipótese de que $\beta>4$ (e a hipótese adicional sobre $k$ quando $\beta=4$ ) no enunciado do teorema abaixo. Ficará claro que esse é o menor expoente que a técnica desenvolvida originalmente pode fornecer.

Teorema 5.5. Se $\beta>4$ e $\phi(t)=\left(q_{1}(t), q_{2}(t), p_{1}(t), p_{2}(t)\right)$ é solução de (5.3) tal que $\lim _{t \rightarrow+\infty}\|\phi\|=0$ então $p_{2}(t)=q_{2}(t)=0$ para todo $t$ suficientemente grande. O mesmo valerá para $\beta=4$ se $0<k<\frac{1}{2^{15} 3^{2}}$.

Demonstração. Já vimos que $p_{2}(\tau)=0$ se e somente se $q_{2}(\tau)=0$ junto com a unicidade de soluções garante que $p_{2}(t)$ e $q_{2}(t)$ são identicamente 
nulos. Assim, podemos trabalhar com $t_{0}=0$ e uma solução assintótica onde $p_{2}\left(t_{0}\right) q_{2}\left(t_{0}\right)<0$. Assuma que $\left(q_{1}, q_{2}, p_{1}, p_{2}\right)(0)=\left(c_{0}, c_{1}, c_{2}, c_{3}\right)$ satisfaz $c_{1}<0<c_{3}$. A demonstração do outro caso é análoga.

Do lema 5.3 temos $\left|p_{2}\right|>\sqrt{k}\left|q_{2}\right|^{\frac{\beta}{2}}$ e de (5.3) $\dot{p_{2}}=k \frac{\beta}{2} \frac{\left|q_{2}\right|^{\beta}}{q_{2}}$. Temos que

$$
\left|\dot{p_{2}}\right|=k \frac{\beta}{2}\left|q_{2}\right|^{\beta-1}=k^{\frac{1}{\beta}} \frac{\beta}{2}\left(\sqrt{k}\left|q_{2}\right|^{\frac{\beta}{2}}\right)^{\frac{2(\beta-1)}{\beta}}<k^{\frac{1}{\beta}} \frac{\beta}{2}\left|p_{2}\right|^{\frac{2(\beta-1)}{\beta}}
$$

pois $\left(\sqrt{k}\left|q_{2}\right|^{\frac{\beta}{2}}\right)>0$ e $\frac{2(\beta-1)}{\beta}>0$.

Estamos supondo que $c_{1}<0<c_{3}$ então temos por (5.3) que $p_{2}>0$ e $\dot{p_{2}}<0$ para todo $t$ em seu domínio de definição, pois para que $p_{2}$ trocasse de sinal preciso que $p_{2}(\tau)=q_{2}(\tau)=0$ para algum $\tau>0$, o que não ocorre. Assim

$$
-\dot{p_{2}}<k^{\frac{1}{\beta}} \frac{\beta}{2} p_{2}^{\frac{2(\beta-1)}{\beta}} \Longrightarrow \frac{-\dot{p_{2}}}{p_{2}^{\frac{2(\beta-1)}{\beta}}}<k^{\frac{1}{\beta}} \frac{\beta}{2}
$$

Do que podemos concluir, usando o lema 4.3.1,

$$
\left(\frac{\beta}{\beta-2}\right) p_{2}^{\frac{-\beta+2}{\beta}}<\left(k^{\frac{1}{\beta}} \frac{\beta}{2}\right) t+\overline{c_{3}} \Longrightarrow p_{2}>\left(k^{\frac{1}{\beta}}\left(\frac{\beta-2}{2}\right) t+\tilde{c_{3}}\right)^{\frac{\beta}{-\beta+2}}>0
$$

para constantes adequadas $\overline{c_{3}}$ e $\tilde{c_{3}}$. Nessa última implicação usamos que $\frac{\beta}{\beta-2}>0$ e que o expoente $\frac{\beta}{-\beta+2}<0$ aplicado para obter a última desigualdade está bem definido (os termos dos dois lados da desigualdade são eventualmente positivos) e inverte nossa desigualdade, pois trata-se de uma função estritamente decrescente.

Analogamente, usando o lema 5.4 temos que $\left|p_{2}\right|<\sqrt{2 k}\left|q_{2}\right|^{\frac{\beta}{2}}$ e como $\dot{p_{2}}=k \frac{\beta}{2} \frac{\left|q_{2}\right|^{\beta}}{q_{2}}$ vale que

$$
\begin{aligned}
&\left|\dot{p_{2}}\right|=k \frac{\beta}{2}\left|q_{2}\right|^{\beta-1}=k \frac{\beta}{2}\left(\frac{1}{\sqrt{2 k} \frac{2(\beta-1)}{\beta}}\right)\left(\sqrt{2 k}\left|q_{2}\right|^{\frac{\beta}{2}}\right)^{\frac{2(\beta-1)}{\beta}}> \\
&>k \frac{\beta}{2}(\sqrt{2 k})^{-\frac{2(\beta-1)}{\beta}}\left|p_{2}\right|^{\frac{2(\beta-1)}{\beta}}
\end{aligned}
$$

Como no raciocínio anterior veja que $p_{2}>0$ e $\dot{p_{2}}<0$, portanto podemos escrever que

$$
\begin{gathered}
\frac{-\dot{p_{2}}}{p_{2} \frac{2(\beta-1)}{\beta}}>k \frac{\beta}{2}(\sqrt{2 k})^{-\frac{2(\beta-1)}{\beta}} \Longrightarrow\left(\frac{\beta}{\beta-2}\right) p_{2}^{\frac{-\beta+2}{\beta}}>k\left(\frac{\beta}{2}\right)(\sqrt{2 k})^{-\frac{2(\beta-1)}{\beta}} t+\overline{c_{3}} \\
\Longrightarrow 0<p_{2}<\left(k\left(\frac{\beta-2}{2}\right)(\sqrt{2 k})^{-\frac{2(\beta-1)}{\beta}} t+\tilde{c_{3}}\right)^{\frac{\beta}{-\beta+2}}
\end{gathered}
$$


Precisamos estimar $p_{1}$. Primeiro, procedemos como acima: pelo lema 5.2 temos $p_{1}>\left(-q_{1}\right)^{\frac{3}{2}}$ e de $\dot{q}_{1}=\left(1+q_{1}\right) p_{1}$ temos que $\dot{q}_{1}>\left(1+q_{1}\right)\left(-q_{1}\right)^{\frac{3}{2}}$. Lembrando que supomos inicialmente $\left|q_{1}\right|<\frac{1}{2}$ e vimos que $q_{1}<0$ podemos concluir que

$$
\dot{q}_{1}>\frac{1}{2}\left(-q_{1}\right)^{\frac{3}{2}} \quad \Longrightarrow \quad 2\left(-q_{1}\right)^{\frac{-1}{2}}>\frac{1}{2} t+\overline{c_{0}}
$$

obtemos, então, usando o lema 4.3.1,

$$
0<-q_{1}<\left(\frac{1}{4} t+\tilde{c_{0}}\right)^{-2}
$$

Olhando para o nível de energia zero (que, como já vimos, é onde uma solução assintótica à origem deve estar) e pelo lema 5.3, veja que $H_{2}-U<0$. Logo,

$$
\frac{\left(1+q_{1}\right)\left(p_{1}{ }^{2}+p_{2}{ }^{2}\right)}{2}+\frac{q_{1}{ }^{3}-k\left|q_{2}\right|^{\beta}}{2}+\frac{k\left|q_{2}\right|^{\beta}-p_{2}^{2}}{2}=\left(1+q_{1}\right) \frac{p_{1}^{2}}{2}+q_{1} \frac{p_{2}^{2}}{2}+\frac{q_{1}^{3}}{2}<0
$$

Dessa forma obtemos que

$$
\frac{p_{1}^{2}}{4}<-q_{1} \frac{p_{2}^{2}}{2}-\frac{q_{1}^{3}}{2}
$$

O que implica, usando as estimativas obtidas acima, que

$$
\frac{p_{1}^{2}}{2}<\left(\frac{1}{4} t+\tilde{c_{0}}\right)^{-2}\left(k\left(\frac{\beta-2}{2}\right)(\sqrt{2 k})^{-\frac{2(\beta-1)}{\beta}} t+\tilde{c_{3}}\right)^{\frac{2 \beta}{-\beta+2}}+\left(\frac{1}{4} t+\tilde{c_{0}}\right)^{-6}
$$

Agora note que, como $k>0$, podemos isolar esse termo obtendo

$$
\left(k\left(\frac{\beta-2}{2}\right)(\sqrt{2 k})^{-\frac{2(\beta-1)}{\beta}} t+\tilde{c_{3}}\right)^{\frac{2 \beta}{-\beta+2}}=\left(k^{\frac{1}{\beta}}\right)^{\frac{2 \beta}{-\beta+2}}\left((2)^{\frac{-\beta+1}{\beta}}\left(\frac{\beta-2}{2}\right) t+\widehat{c_{3}}\right)^{\frac{2 \beta}{-\beta+2}}
$$

Veja então que $2^{\frac{-\beta+1}{\beta}}\left(\frac{\beta-2}{2}\right) \geq \frac{1}{2}>\frac{1}{4}$ pois, como $\beta \geq 4$, temos que $\frac{\beta-2}{2} \geq 1$ e como $\frac{-\beta+1}{\beta}=-1+\frac{1}{\beta}>-1$ temos $(2)^{\frac{-\beta+1}{\beta}}>\frac{1}{2}$. Portanto, pelo lema 4.4.2, existe $t_{1}$ suficientemente grande de forma que podemos escrever, para todo $t>\max \left\{0, t_{1}\right\}$,

$$
\left((2)^{\frac{-\beta+1}{\beta}}\left(\frac{\beta-2}{2}\right) t+\widehat{c_{3}}\right)^{\frac{2 \beta}{-\beta+2}}<\left(\frac{1}{4} t+\tilde{c_{0}}\right)^{\frac{2 \beta}{-\beta+2}}
$$

e obtemos desta forma que

$$
\frac{p_{1}^{2}}{2}<\left(k^{\frac{1}{\beta}}\right)^{\frac{2 \beta}{-\beta+2}}\left(\frac{1}{4} t+\tilde{c_{0}}\right)^{-2}\left((2)^{\frac{-\beta+1}{\beta}}\left(\frac{\beta-2}{2}\right) t+\widehat{c_{3}}\right)^{\frac{2 \beta}{-\beta+2}}+\left(\frac{1}{4} t+\tilde{c_{0}}\right)^{-6}<
$$




$$
<\left(k^{\frac{1}{\beta}}\right)^{\frac{2 \beta}{-\beta+2}}\left(\frac{1}{4} t+\tilde{c_{0}}\right)^{\frac{4(\beta-1)}{-\beta+2}}+\left(\frac{1}{4} t+\tilde{c_{0}}\right)^{-6}
$$

$\mathrm{Na}$ expressão acima tomando $t_{2}$ suficientemente grande e $\beta>2$ tal que $\frac{4(\beta-1)}{-\beta+2} \geq-6$ (ou seja, que $\beta \geq 4$ conforme foi pedido nas hipóteses enunciadas) podemos utilizar os lemas 4.4.1 e 4.4.2 no termo da direita para obter

$$
\frac{p_{1}^{2}}{2}<\left(1+\left(k^{\frac{1}{\beta}}\right)^{\frac{2 \beta}{-\beta+2}}\right)\left(\frac{1}{4} t+\tilde{c_{0}}\right)^{\frac{4(\beta-1)}{-\beta+2}}
$$

Assim temos, finalmente, que

$$
p_{1}<\sqrt{2\left(1+k^{\frac{2}{-\beta+2}}\right)}\left(\frac{1}{4} t+\tilde{c_{0}}\right)^{\frac{2(\beta-1)}{-\beta+2}}
$$

Agora estamos em condições de completar a demonstração. Defina para $t>\max \left\{1, t_{1}, t_{2}\right\}$

$$
f(t)=\int_{t}^{\infty} \frac{p_{2}^{2}(s)}{2} d s
$$

Note também que $\dot{p_{1}}<-\frac{p_{2}^{2}}{2}$. Assim, vale que

$$
\lim _{s \rightarrow \infty} p_{1}(s)=p_{1}(t)+\int_{t}^{\infty} \dot{p_{1}}(w) d w<p_{1}(t)-f(t)
$$

Como

$$
\begin{gathered}
f(t)=\int_{t}^{\infty} \frac{p_{2}^{2}(s)}{2}>\frac{1}{2} \int_{t}^{\infty}\left(k^{\frac{1}{\beta}}\left(\frac{\beta-2}{2}\right) s+\tilde{c_{3}}\right)^{\frac{2 \beta}{-\beta+2}}= \\
=\left.\left(\frac{-1}{k^{\frac{1}{\beta}}(\beta+2)}\left(k^{\frac{1}{\beta}}\left(\frac{\beta-2}{2}\right) s+\tilde{c_{3}}\right)^{\frac{\beta+2}{-\beta+2}}\right)\right|_{t} ^{\infty} \\
=\frac{1}{k^{\frac{1}{\beta}}(\beta+2)}\left(k^{\frac{1}{\beta}}\left(\frac{\beta-2}{2}\right) t+\tilde{c_{3}}\right)^{\frac{\beta+2}{-\beta+2}}
\end{gathered}
$$

temos que

$$
\lim _{s \rightarrow \infty} p_{1}(s)<\sqrt{2\left(1+k^{-\beta+2}\right)}\left(\frac{1}{4} t+\tilde{c_{0}}\right)^{\frac{2(\beta-1)}{-\beta+2}}-\frac{1}{k^{\frac{1}{\beta}}(\beta+2)}\left(k^{\frac{1}{\beta}}\left(\frac{\beta-2}{2}\right) t+\tilde{c_{3}}\right)^{\frac{\beta+2}{-\beta+2}}
$$

Se tivermos que $\frac{2(\beta-1)}{-\beta+2}<\frac{\beta+2}{-\beta+2}$ ou seja, como $-\beta+2<0$, que

$$
2(\beta-1)>\beta+2 \Longrightarrow \beta>4
$$


podemos majorar, para algum $t_{3}$ suficientemente grande, passando os termos externos para dentro de seus respectivos parêntesis e olhando apenas para o maior expoente (lema 4.4.1). Assim, para $t>\max \left\{1, t_{1}, t_{2}, t_{3}\right\}$ temos que

$$
\begin{gathered}
\lim _{s \rightarrow \infty} p_{1}(s)<\frac{1}{k^{\frac{1}{\beta}}(\beta+2)}\left(k^{\frac{1}{\beta}}\left(\frac{\beta-2}{2}\right) t+\tilde{c_{3}}\right)^{\frac{\beta+2}{-\beta+2}}+\ldots \\
\ldots-\frac{1}{k^{\frac{1}{\beta}}(\beta+2)}\left(k^{\frac{1}{\beta}}\left(\frac{\beta-2}{2}\right) t+\tilde{c_{3}}\right)^{\frac{\beta+2}{-\beta+2}}=0
\end{gathered}
$$

o que é uma clara contradição, pois supomos que $\phi(t)$ satisfazia $\lim _{t \rightarrow+\infty}\|\phi(t)\|=$ 0 .

Em particular, podemos melhorar esse resultado utilizando o lema 4.4.2, i.e., podemos incluir o caso $\beta=4$ se $k>0$ for escolhido de forma que o fator que multiplica $t$ no primeiro termo seja maior do que o fator que multiplica $t$ no segundo termo, ou seja, de forma que

$$
\left(\frac{1}{6 k^{\frac{1}{4}}}\right)^{-\frac{1}{3}} k^{\frac{1}{4}}<\frac{1}{4}{\sqrt{2\left(1+k^{-1}\right)}}^{-\frac{1}{3}}
$$

(veja que mandamos os termos externos para dentro dos parêntesis e calculamos apenas para $\beta=4$ ). As contas são longas e não acrescentam muito nesta demonstração, por isso elas estão feitas no apêndice onde podem ser estudadas de forma independente pelos interessados.

Corolário 5.6. Dado $\beta>4$ e $k>0$ a dimensão da bacia de atração da origem para as equações de $H_{1}$ e $H_{2}$ não é igual. O mesmo ocorre para $\beta=4$ se $k$ for escolhido satisfazendo $0<k<\frac{1}{2^{15} 3^{2}}$

Demonstração. É uma simples consequência do lema 5.1 que $B A_{1}$ é uma subvariedade de $\mathbb{R}^{4}$ de dimensão 2 (pois $q_{1}=f\left(p_{1}\right)$ e $q_{2}=g\left(p_{2}\right)$ ).

Por sua vez o teorema 5.5 diz que, sob as hipóteses acima, uma solução para $H_{2}$ assintótica à origem deve satisfazer $p_{2}(t)=q_{2}(t)=0$ para todo $t$. Assim a dimensão da bacia de atração da origem para $H_{2}$ é 1 .

Portanto as dimensões das bacias de atração são diferentes sempre que respeitadas as condições do teorema 5.5.

Em particular, o resultado principal deste capítulo é que o menor expoente $\beta$ que poderia ter sido usado no artigo original era $\beta=6$ (lembrando que no original tinhamos $k=1$ e $\beta$ um número inteiro par). Note que não discutimos o caso $\beta=2$, mas ele certamente não poderia ter sido estudado usando diretamente este raciocínio. Concluímos também que o resultado obtido no artigo original pode ser generalizado para todo $\beta$ inteiro par maior ou igual a 6 . 
Em particular, para a família proposta nesta dissertação temos que a constante $k>0$ incluida na energia potencial pode ser tomanda suficientemente pequena para concluirmos o mesmo para $\beta=4$. No próximo capítulo faremos outra modificação nos expoentes originais que permitirá um resultado mais profundo, ainda baseado nas idéias originais. 


\section{Capítulo 6}

\section{Um critério explícito}

Neste capítulo generalizamos o resultado do capítulo anterior trocando o termo $q_{1}^{3}$ na energia potencial por um termo da forma $q_{1}\left|q_{1}\right|^{\alpha}$ para $\alpha \in \mathbb{R}$ $\operatorname{com} \alpha>1$. Isso garante a existência de trajetória assintótica à origem para o novo $H_{1}$ pelo mesmo raciocínio feito anteriormente, já que 0 é um ponto de sela para $q_{1}\left|q_{1}\right|^{\alpha}$ quando $\alpha$ é positivo. Portanto o objetivo aqui é estudar quais hipóteses técnicas precisamos para garantir o resultado desejado usando basicamente o mesmo raciocínio do capítulo anterior, obtendo assim uma classe mais ampla de exemplos onde a dimensão da bacia de atração da origem é influenciada pela energia cinética.

Com o intuito de evitar uma notação mais "pesada" mantivemos os mesmos nomes para todas as variáveis. A estrutura da demonstração também foi mantida em sua íntegra, na esperança que essas duas decisões simplifiquem a leitura desse capítulo. Além disso os leitores interessados ficam convidados a comparar esse capítulo ao anterior apenas substituindo $\alpha$ por 2 .

Para $k>0$ e dados $\alpha, \beta \in \mathbb{R}$ satisfazendo $\alpha>1$ e $\beta>2$ considere

$$
\begin{gathered}
\pi: \Omega=\left\{\left(q_{1}, q_{2}\right) \in \mathbb{R}^{2}: q_{1}>-1\right\} \rightarrow \mathbb{R} \\
\pi\left(q_{1}, q_{2}\right)=\frac{q_{1}\left|q_{1}\right|^{\alpha}-k\left|q_{2}\right|^{\beta}}{2}
\end{gathered}
$$

Considere também

$$
\begin{gathered}
T_{1}, T_{2}: \Omega \times \mathbb{R}^{2} \rightarrow \mathbb{R}^{+} \\
T_{1}=\frac{p_{1}{ }^{2}+p_{2}{ }^{2}}{2}, \quad T_{2}=\frac{\left(1+q_{1}\right)\left(p_{1}{ }^{2}+p_{2}{ }^{2}\right)}{2}
\end{gathered}
$$

Se definirmos $H_{1}=T_{1}+\pi$, obtemos que

$$
H_{1}\left(q_{1}, q_{2}, p_{1}, p_{2}\right)=\frac{p_{1}^{2}+p_{2}^{2}}{2}+\frac{q_{1}\left|q_{1}\right|^{\alpha}-k\left|q_{2}\right|^{\beta}}{2}
$$


Cujas equações hamiltonianas são,

$$
\left\{\begin{array}{cc}
\dot{q_{1}}=p_{1} & \dot{q}_{2}=p_{2} \\
\dot{p_{1}}=-\frac{(1+\alpha)}{2}\left|q_{1}\right|^{\alpha} & \dot{p_{2}}=k \frac{\beta}{2} \frac{\left|q_{2}\right|^{\beta}}{q_{2}}
\end{array}\right.
$$

O lema 4.1.1 garante que este sistema está bem definido e é $C^{1}\left(\mathbb{R}^{4}\right)$. Assim, as duas funções são diferenciáveis em todo ponto do domínio. Em particular, para condições iniciais adequadas, $\ddot{q}_{1}=-\frac{(1+\alpha)}{2}\left|q_{1}\right|^{\alpha}$ e $\ddot{q}_{2}=k \frac{\beta}{2} \frac{\left|q_{2}\right|^{\beta}}{q_{2}}$ tem como possíveis soluções

$$
\left\{\begin{array}{l}
q_{1}(t)=-\left(\frac{4}{(\alpha-1)^{2}}\right)^{\frac{1}{\alpha-1}}\left|t+c_{0}\right|^{\frac{-2}{\alpha-1}} \\
q_{2}(t)=\left(\frac{4}{k(\beta-2)^{2}}\right)^{\frac{1}{\beta-2}}\left|t+c_{1}\right|^{\frac{-2}{\beta-2}}
\end{array}\right.
$$

para $t>\max \left\{-c_{0},-c_{1}\right\}$, ambas assintóticas à origem. Note mais uma vez que o caso $\beta=2$ não será contemplado, já que possui soluções assintóticas à origem do tipo exponencial. Contudo, é fácil ver que o lema abaixo ainda vale neste caso.

A análise da bacia de atração do sistema (6.1) será simplificada se definirmos as funções

$H_{1,1}\left(q_{1}(t), p_{1}(t)\right)=\frac{p_{1}^{2}(t)}{2}+\frac{q_{1}(t)\left|q_{1}(t)\right|^{\alpha}}{2}, H_{1,2}\left(q_{2}(t), p_{2}(t)\right)=\frac{p_{2}^{2}(t)}{2}+\frac{-k\left|q_{2}(t)\right|^{\beta}}{2}$

Lema 6.1. A bacia de atração da origem para o sistema (6.1) é o conjunto

$$
\begin{gathered}
B A_{1}=\left\{\left(q_{1}, q_{2}, p_{1}, p_{2}\right) \in \Omega \times \mathbb{R}^{2}: H_{1,1}\left(q_{1}, p_{1}\right)=0=H_{1,2}\left(q_{2}, p_{2}\right),\right. \\
\left.p_{1} q_{1} \leq 0 \text { e } p_{2} q_{2} \leq 0\right\}
\end{gathered}
$$

Demonstração. Seja $\left(q_{1}, q_{2}, p_{1}, p_{2}\right)(t)$ uma solução de (6.1) assintótica à origem e $I$ seu intervalo maximal de definição. Temos que

$$
H_{1}\left(q_{1}(t), q_{2}(t), p_{1}(t), p_{2}(t)\right)=0, \quad \forall t \in I
$$

pois $H_{1}$ é contínua, constante sobre as soluções e $H_{1}(0,0,0,0)=0$. Portanto, como temos $H_{1}=H_{1,1}+H_{1,2}=0$ para todo $t$, vale que $H_{1,1}\left(q_{1}, p_{1}\right)=$ $-H_{1,2}\left(q_{2}, p_{2}\right)$ Um cálculo simples mostra que uma solução de (6.1) satisfaz $\dot{H}_{1,1}=\dot{H}_{1,2}=0$. Além disso $\lim _{t / \infty} H_{1,1}\left(q_{1}(t), p_{1}(t)\right)=0$ pois $\left(q_{1}, q_{2}, p_{1}, p_{2}\right)(t)$ é uma solução de (6.1) assintótica à origem. Note então que, como acima, essas observações implicam que $H_{1,1}\left(q_{1}, p_{1}\right)=0=H_{1,2}\left(q_{2}, p_{2}\right)$

Além disso se uma solução é assintótica à origem, como $\left(p_{2} q_{2}\right)=k \frac{\beta}{2}\left|q_{2}\right|^{\beta}+$ $p_{2}^{2} \geq 0$, a função $p_{2}(t) q_{2}(t)$ é crescente para todo $t$ e deve tender a zero. Logo 
$p_{2} q_{2} \leq 0$. Analogamente note que $H_{1,1}=0$ implica que $q_{1} \leq 0$ que por sua vez garante que $\left(p_{1} q_{1}\right)=\frac{-(1+\alpha)}{2} q_{1}\left|q_{1}\right|^{\alpha}+p_{1}^{2} \geq 0$. Portanto $p_{1} q_{1} \leq 0$.

Por outro lado considere uma solução passando por $\left(q_{1}, q_{2}, p_{1}, p_{2}\right) \in \Omega \times$ $\mathbb{R}^{2}$ e tal que $H_{1,1}\left(q_{1}, p_{1}\right)=H_{1,2}\left(q_{2}, p_{2}\right)=0, p_{1} q_{1} \leq 0, p_{2} q_{2} \leq 0$. Quero mostrar que ela é assintótica à origem. Como $H_{1}=H_{1,1}+H_{1,2}$ temos que $H_{1}=0$. Note também que $H_{1,1}\left(q_{1}, p_{1}\right)=0$ mostra que $q_{1}=0$ se e só se $p_{1}=0$ e de forma análoga conclua que $p_{2}=0$ se e só se $q_{2}=0$.

Temos então alguns casos para estudar:

1. O caso $(0,0,0,0)$ é trivial, pois a trajetória $(0,0,0,0)$ é a única solução assintótica à origem.

2. Para os casos $\left(q_{1}(0), 0, p_{1}(0), 0\right)$ onde $p_{1}(0) q_{1}(0)<0$ e $\left(0, q_{2}(0), 0, p_{2}(0)\right)$ e $p_{2}(0) q_{2}(0)<0$ note que os planos $(X, 0, Y, 0)$ e $(0, X, 0, Y)$ são invariantes pelas soluções de (6.1). Podemos utilizar então as soluções encontradas na seção 4.2 para construir uma solução assintótica à origem da forma $\left(q_{1}(t), 0, p_{1}(t), 0\right)$ ou $\left(0, q_{2}(t), 0, p_{2}(t)\right)$ conforme o caso estudado.

3. Finalmente, para o caso em que $q_{1}(0) p_{1}(0)<0$ e $q_{2}(0) p_{2}(0)<0$, construímos uma solução assintótica à origem a partir das soluções para $H_{1,1}$ e $H_{1,2}=0$ encontradas na seção 4.2.

Assim, como cada conjunto está contido no outro, obtivemos sua igualdade.

Façamos agora a analise de $H_{2}=T_{2}+\pi$

$$
H_{2}\left(q_{1}, q_{2}, p_{1}, p_{2}\right)=\frac{\left(1+q_{1}\right)\left(p_{1}^{2}+p_{2}^{2}\right)}{2}+\frac{q_{1}\left|q_{1}\right|^{\alpha}-k\left|q_{2}\right|^{\beta}}{2}
$$

Cujas equações hamiltonianas são,

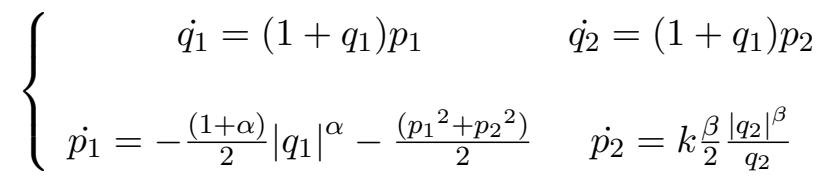

Novamente, o lema 4.1.1 garantirá que este sistema está bem definido e é $C^{1}\left(\mathbb{R}^{4}\right)$. Isso, em particular, quer dizer que se tomarmos condições iniciais $\left(q_{1}, q_{2}, p_{1}, p_{2}\right)(0)$ tais que $p_{2}(0)=q_{2}(0)=0$ teremos $p_{2}(t)=q_{2}(t)=0$ para todo $t$, o que pode ser verificado por substituição e usando a unicidade das soluções. Portanto, as equações para $\dot{p_{1}}$ e $\dot{q}_{1}$ sobre uma solução satisfazendo essa condição inicial são

$$
\dot{p_{1}}=-\frac{(1+\alpha)}{2}\left|q_{1}\right|^{\alpha}-\frac{1}{2} p_{1}^{2}, \quad \dot{q_{1}}=\left(1+q_{1}\right) p_{1}
$$


e, portanto $\left(q_{1}, p_{1}\right)$ satisfazem as equações hamiltonianas para $\tilde{H}_{2}\left(q_{1}, p_{1}\right)=$ $\left(1+q_{1}\right) \frac{p_{1}^{2}}{2}+\frac{q_{1}\left|q_{1}\right|^{\alpha}}{2}$. Como a energia potencial (unidimensional) associada a esse sistema tem ponto de sela na origem note que as equações hamiltonianas de $\tilde{H}_{2}$ possuem uma trajetória assintótica à origem.

No que segue, o objetivo é mostrar que a condição $p_{2}(t)=q_{2}(t)=0$, válida para todo $t$ onde uma solução estiver definida, é necessária para que uma solução de (6.3) seja assintótica à origem.

Vamos começar com poucas observações sobre a natureza das soluções assintóticas à origem para esse sistema em particular. Seja então $\phi(t)=$ $\left(q_{1}(t), q_{2}(t), p_{1}(t), p_{2}(t)\right)$ solução de $(6.3)$ tal que $\lim _{t \rightarrow+\infty}\|\phi(t)\|=0$. Estamos estudando apenas as trajetórias assintóticas, então podemos considerar o tempo $t_{0}$ tal que, para todo $t>t_{0},\left|q_{1}(t)\right|<\frac{1}{2}$ (assumimos $t_{0}=0 \mathrm{sem}$ perda de generalidade). Veja que, como $\alpha>1$

$$
\dot{p_{1}}=-\frac{(1+\alpha)}{2}\left|q_{1}\right|^{\alpha}-\frac{\left(p_{1}^{2}+p_{2}^{2}\right)}{2} \leq 0
$$

i.e., $p_{1}$ é não-crescente. Então, para que a solução seja assintótica, só podemos ter que $p_{1}(t) \geq 0$, para todo $t \in \mathbb{R}$, já que $\lim _{t \rightarrow+\infty}\|\phi(t)\|=0$. Assim

$$
\dot{q}_{1}=\left(1+q_{1}\right) p_{1} \geq \frac{1}{2} p_{1} \geq 0
$$

i.e., $q_{1}$ é não-decrescente e portanto para uma trajetória assintótica temos necessariamente que $q_{1}(t) \leq 0$, para todo $t>0$.

Note, usando $(6.3)$, que $\dot{p}_{1}(0)=0$ se e somente se $q_{1}(0)=p_{1}(0)=$ $p_{2}(0)=0$. Sobre uma trajetória assintótica $q_{1}(t)$ é não-decrescente e tende zero, portanto $q_{1}(t)=0$ para todo $t>0$ e como nessa trajetória $p_{1}(t)$ é nãocrescente e tende zero também temos que $p_{1}(t)=0$ para todo $t>0$. Desta forma $-\frac{p_{2}^{2}(t)}{2}=0$ para todo $t>0$ e assim temos que $\dot{q}_{2}=\left(1+q_{1}\right) p_{2}=0$, ou seja, para que a trajetória seja assintótica à origem precisamos ter $q_{2}(t)=0$ para todo $t>0$. Desta forma mostramos que se $\dot{p_{1}}(0)=0$ então a solução $\phi(s)$ será constante e igual ao ponto $(0,0,0,0)$, para todo $s>0$.

No interesse de considerar apenas as soluções assintóticas à origem com $p_{2}(s), q_{2}(s) \neq 0$ para algum $s>0$, podemos considerar, daqui em diante $p_{1}(t)>0$ para todo $t \in \mathbb{R}^{+}$e, consequentemente, $q_{1}(t)<0$ para todo $t \in \mathbb{R}^{+}$.

Desta forma, para uma trajetória assintótica e para todo $t>0$ temos que $0>q_{1}(t)>-\frac{1}{2}$. Então, como $k>0$

$$
\left(\dot{q_{2} p_{2}}\right)=\left(1+q_{1}\right) p_{2}^{2}+k \frac{\beta}{2}\left|q_{2}\right|^{\beta} \geq \frac{1}{2} p_{2}^{2}+k \frac{\beta}{2}\left|q_{2}\right|^{\beta} \geq 0
$$

Concluímos portanto que $q_{2}(t) p_{2}(t) \leq 0$, pois $\lim _{t \rightarrow+\infty}\|\phi(t)\|=0$. Mais ainda, se olharmos para as equações

$$
\left\{\begin{array}{c}
\dot{q_{2}}=\left(1+q_{1}\right) p_{2} \\
\dot{p_{2}}=k \frac{\beta}{2} \frac{\left|q_{2}\right|^{\beta}}{q_{2}}
\end{array}\right.
$$


podemos ver que $p_{2}(\tau)=0$ se e somente se $q_{2}(\tau)=0$, para algum $\tau \in$ $\mathbb{R}$. De fato, se $p_{2}(\tau)=0$ e $q_{2}(\tau)>0$ temos que $\dot{p_{2}}(\tau)>0$, i.e., $p_{2}(\tau)$ é não-decrescente. Assim a continuidade das equações deixa afirmar que teríamos um intervalo $] \tau, \tau_{1}\left[\right.$ em que $p_{2}>0$ e $q_{2}>0$, o que contradiz $q_{2}(t) p_{2}(t) \leq 0$. O caso $p_{2}(\tau)=0$ e $q_{2}(\tau)<0$ é análogo, assim como os dois restantes. Se tomarmos condições iniciais $\left(q_{1}(0), q_{2}(0), p_{1}(0), p_{2}(0)\right)$ tais que $p_{2}(0)=q_{2}(0)=0$ teremos $p_{2}(t)=q_{2}(t)=0$ usando a unicidade de soluções. Desta forma podemos supor sem perda de generalidade que $q_{2}(t) p_{2}(t)<0$ para todo $t>0$, pois não estamos interessados nas soluções que satisfazem $p_{2}(t)=q_{2}(t)=0$ para todo $t \in \mathbb{R}$.

Passamos agora para três resultados intermediários que utilizam as informações obtidas nos últimos parágrafos e funções auxiliares para comparar o comportamento entre as funções coordenadas de soluções que sejam assintóticas à origem. Note que esses resultados e suas respectivas demonstrações estão fortemente relacionadas com o segundo lema de comparação que apresentamos no capítulo 4 (lema 4.3.2).

Lema 6.2. Se $\phi(t)$ é solução de (6.3) tal que $\lim _{t \rightarrow+\infty}\|\phi(t)\|=0$ então para todo $t>0$ temos $p_{1}(t)>\left(-q_{1}(t)\right)^{\frac{\alpha+1}{2}}$

Demonstração. Defina $V\left(q_{1}(t), p_{1}(t)\right)=-\frac{1}{2}\left|q_{1}(t)\right|^{\alpha+1}+\frac{1}{2} p_{1}^{2}(t)$. Como discutimos acima, as hipóteses implicam que $q_{1}(t)<0$ para todo $t>0$. Assim, como

$$
\frac{d}{d t}\left|q_{1}(t)\right|^{\alpha+1}=(\alpha+1) \frac{\left|q_{1}(t)\right|^{\alpha+1}}{q_{1}(t)}=-(\alpha+1)\left|q_{1}(t)\right|^{\alpha}
$$

Calculamos

$$
\begin{aligned}
\dot{V}\left(q_{1}, p_{1}\right) & =-\frac{(\alpha+1)}{2} \frac{\left|q_{1}\right|^{\alpha+1}}{q_{1}} \dot{q}_{1}+\frac{1}{2} p_{1} \dot{p_{1}}= \\
& =\frac{(\alpha+1)}{2}\left|q_{1}\right|^{\alpha}\left(1+q_{1}\right) p_{1}+\frac{1}{2} p_{1}\left(-(1+\alpha)\left|q_{1}\right|^{\alpha}-p_{1}^{2}-p_{2}^{2}\right)= \\
& =\frac{p_{1}}{2}\left((\alpha+1) q_{1}\left|q_{1}\right|^{\alpha}-p_{1}^{2}-p_{2}^{2}\right)<0
\end{aligned}
$$

pois vimos que $p_{1}<0$. Desta forma obtemos (já que $\lim _{t \rightarrow+\infty}\|\phi(t)\|=0$ ) que $V\left(q_{1}(t), p_{1}(t)\right)>0$. Assim $p_{1}^{2}(t)>\left|q_{1}(t)\right|^{\alpha+1}$ e como $p_{1}(t)>0$ e $q_{1}(t)<0$ para todo $t>0$ temos $p_{1}(t)>\left(-q_{1}(t)\right)^{\frac{\alpha+1}{2}}$

Lema 6.3. Se $\phi(t)$ é solução de (6.3) tal que $\lim _{t \rightarrow+\infty}\|\phi(t)\|=0$ e $p_{2}(\tau) q_{2}(\tau) \neq$ 0 para algum $\tau>0$ então para todo $t>0$ temos $\left|p_{2}(t)\right|>\sqrt{k}\left|q_{2}(t)\right|^{\frac{\beta}{2}}$

Demonstração. Defina $U\left(q_{2}(t), p_{2}(t)\right)=-k \frac{1}{2}\left|q_{2}(t)\right|^{\beta}+\frac{1}{2} p_{2}^{2}(t)$. Temos que 


$$
\dot{U}\left(q_{2}, p_{2}\right)=-k \frac{\beta}{2} \frac{\left|q_{2}\right|^{\beta}}{q_{2}}\left(1+q_{1}\right) p_{2}+p_{2}\left(k \frac{\beta}{2} \frac{\left|q_{2}\right|^{\beta}}{q_{2}}\right)=-k \frac{\beta}{2} \frac{\left|q_{2}\right|^{\beta}}{q_{2}} q_{1} p_{2}<0
$$

A ultima desigualdade segue da seguinte forma: sabemos que $q_{1}(t)<0$ para todo $t>0$ e que $p_{2}(\tau) q_{2}(\tau)<0$. Desta forma $p_{2}(t) q_{2}(t) \neq 0$ para todo $t$ (como já argumentamos anteriormente). Além disso como $q_{2}(t) p_{2}(t)<0$ podemos dividir por $q_{2}^{2}(t)$ para concluir que $\frac{p_{2}(t)}{q_{2}(t)}<0$. Desta forma as hipóteses implicam que $U\left(q_{2}(t), p_{2}(t)\right)>0$, logo $\left|p_{2}(t)\right|>\sqrt{k}\left|q_{2}(t)\right|^{\frac{\beta}{2}}$

Lema 6.4. Se $\phi(t)$ é solução de (6.3) tal que $\lim _{t \rightarrow+\infty}\|\phi(t)\|=0$ e $p_{2}(\tau) q_{2}(\tau) \neq$ 0 para algum $\tau>0$ então para todo $t>0$ temos $\left|p_{2}(t)\right|<\sqrt{2 k}\left|q_{2}(t)\right|^{\frac{\beta}{2}}$

Demonstração. Defina $U_{2}\left(q_{1}(t), q_{2}(t), p_{2}(t)\right)=\left(1+q_{1}(t)\right) \frac{p_{2}^{2}(t)}{2}-k \frac{\left|q_{2}(t)\right|^{\beta}}{2}$. Veja que

$$
\begin{aligned}
\dot{U}_{2}\left(q_{1}, q_{2}, p_{2}\right) & =\frac{p_{2}^{2}}{2} \dot{q}_{1}+\left(1+q_{1}\right) p_{2} \dot{p_{2}}-k \frac{\beta}{2} \frac{\left|q_{2}\right|^{\beta}}{q_{2}} \dot{q_{2}}= \\
& =\frac{p_{2}^{2}}{2}\left(1+q_{1}\right) p_{1}+\left(1+q_{1}\right) p_{2}\left(\frac{k \beta}{2} \frac{\left|q_{2}\right|^{\beta}}{q_{2}}\right)-\frac{k \beta}{2} \frac{\left|q_{2}\right|^{\beta}}{q_{2}}\left(1+q_{1}\right) p_{2}= \\
& =\frac{p_{2}^{2}}{2}\left(1+q_{1}\right) p_{1} \geq 0
\end{aligned}
$$

pois $\left|q_{1}(t)\right|<\frac{1}{2}$ e $p_{1}(t)>0$, para todo $t>0$. Como vimos $p_{2}(\tau)=0$ se e somente se $q_{2}(\tau)=0$, logo $U_{2} \neq 0$, pois teríamos como antes que $p_{2}(t) q_{2}(t)=0$. Além disso, por hipótese $\lim _{t \rightarrow+\infty}\|\phi(t)\|=0$, então temos que $U_{2}\left(q_{1}(t), q_{2}(t), p_{2}(t)\right)<0$ para todo $t>0$ e, como $\left|q_{1}(t)\right|<\frac{1}{2}$ e $\dot{q}_{1}(t)>0$ para todo $t>0$, temos

$$
\left(1+q_{1}\right) \frac{p_{2}^{2}}{2}<k \frac{\left|q_{2}\right|^{\beta}}{2} \Longrightarrow p_{2}^{2}<2 k\left|q_{2}\right|^{\beta}
$$

ou seja, $\left|p_{2}(t)\right|<\sqrt{2 k}\left|q_{2}(t)\right|^{\frac{\beta}{2}}$

Procedemos com o intuito de mostrar que a condição $p_{2}(t)=q_{2}(t)=0$ para algum $t>0$ é necessária para que uma solução de (6.3) seja assintótica à origem. Até agora só precisamos de $\alpha>1$ e $\beta>2$ como condições de regularidade (lema 4.1.1). Infelizmente as contas abaixo exigem um pouco mais do que isso. Por razões de objetividade incluímos as hipóteses técnicas no enunciado do teorema abaixo, contudo a demonstração abaixo foi preparada de forma que o leitor possa examinar também a forma como essas suposições foram escolhidas. Discutiremos melhor essas hipóteses nos corolários que aparecem na sequência dessa demonstração. 
Teorema 6.5. Suponha que $k>0, \alpha>1, \beta>2$ e $\phi(t)=\left(q_{1}, q_{2}, p_{1}, p_{2}\right)(t)$ é solução de (6.3) tal que $\lim _{t \rightarrow+\infty}\|\phi(t)\|=0$. Se tivermos que

$$
\frac{-\alpha}{\alpha-1} \leq \frac{\beta}{-\beta+2} \quad e \quad \frac{-1}{\alpha-1}<\frac{2}{-\beta+2}
$$

então $p_{2}(t)=q_{2}(t)=0$ para todo $t$. Se supormos adicionalmente que

$k<\left((\beta+2)^{2}\left(\frac{2(\beta-2)}{\alpha-1}\right)^{\frac{-2(\beta+2)}{-\beta+2}}\left(1+\sqrt{1+2\left(\frac{1}{\beta+2}\right)^{2}\left(\frac{2(\beta-2)}{\alpha-1}\right)^{\frac{2(\beta+2)}{-\beta+2}}}\right)\right)^{\frac{-\beta+2}{2}}$

a segunda hipótese acima pode ser enfraquecida para $\frac{-1}{\alpha-1} \leq \frac{2}{-\beta+2}$.

Demonstração. Já vimos que $p_{2}(\tau)=0$ se e somente se $q_{2}(\tau)=0$ junto com a unicidade de soluções garante que $p_{2}(t)$ e $q_{2}(t)$ são identicamente nulos. Assim, podemos trabalhar com $t_{0}=0$ e uma solução assintótica onde $p_{2}\left(t_{0}\right) q_{2}\left(t_{0}\right)<0$. Assuma que $\left(q_{1}, q_{2}, p_{1}, p_{2}\right)(0)=\left(c_{0}, c_{1}, c_{2}, c_{3}\right)$ satisfaz $c_{1}<0<c_{3}$. A demonstração do outro caso é análoga.

Do lema 6.3 temos $\left|p_{2}\right|>\sqrt{k}\left|q_{2}\right|^{\frac{\beta}{2}}$ e como $\dot{p_{2}}=k \frac{\beta}{2} \frac{\left|q_{2}\right|^{\beta}}{q_{2}}$, temos que

$$
\left|\dot{p_{2}}\right|=k \frac{\beta}{2}\left|q_{2}\right|^{\beta-1}=k^{\frac{1}{\beta}} \frac{\beta}{2}\left(\sqrt{k}\left|q_{2}\right|^{\frac{\beta}{2}}\right)^{\frac{2(\beta-1)}{\beta}}<k^{\frac{1}{\beta}} \frac{\beta}{2}\left|p_{2}\right|^{\frac{2(\beta-1)}{\beta}}
$$

pois $\left(\sqrt{k}\left|q_{2}\right|^{\frac{\beta}{2}}\right)>0$ e $\frac{2(\beta-1)}{\beta}>0$.

Estamos supondo que $c_{1}<0<c_{3}$ então temos por (6.3) que $p_{2}>0$ e $\dot{p_{2}}<0$ para todo $t$ em seu domínio de definição, pois para que $p_{2}$ trocasse de sinal preciso que $p_{2}(\tau)=q_{2}(\tau)=0$ para algum $\tau>0$, o que não ocorre. Assim

$$
-\dot{p_{2}}<k^{\frac{1}{\beta}} \frac{\beta}{2} p_{2}^{\frac{2(\beta-1)}{\beta}} \Longrightarrow \frac{-\dot{p_{2}}}{p_{2}^{\frac{2(\beta-1)}{\beta}}}<k^{\frac{1}{\beta}} \frac{\beta}{2}
$$

Do que podemos concluir

$$
\left(\frac{\beta}{\beta-2}\right) p_{2}^{\frac{-\beta+2}{\beta}}<k^{\frac{1}{\beta}}\left(\frac{\beta}{2}\right) t+\overline{c_{3}} \Longrightarrow p_{2}>\left(k^{\frac{1}{\beta}}\left(\frac{\beta-2}{2}\right) t+\tilde{c_{3}}\right)^{\frac{\beta}{-\beta+2}}>0
$$

para constantes adequadas $\overline{c_{3}}$ e $\tilde{c_{3}}$. Nessa última implicação, como $\beta>2$, usamos que $\frac{\beta}{\beta-2}>0$ e que o expoente $\frac{\beta}{-\beta+2}<0$ aplicado para obter a última desigualdade está bem definido (ambos termos são positivos) e inverte nossa desigualdade, pois se trata de uma função estritamente decrescente.

Analogamente, usando o lema 6.4 temos que $\left|p_{2}\right|<\sqrt{2 k}\left|q_{2}\right|^{\frac{\beta}{2}}$ e $\dot{p_{2}}=$ $k \frac{\beta}{2} \frac{\left|q_{2}\right|^{\beta}}{q_{2}}$ resultam que

$$
\left|\dot{p_{2}}\right|=k \frac{\beta}{2}\left|q_{2}\right|^{\beta-1}=k \frac{\beta}{2}\left(\frac{1}{\sqrt{2 k} \frac{2(\beta-1)}{\beta}}\right)\left(\sqrt{2 k}\left|q_{2}\right|^{\frac{\beta}{2}}\right)^{\frac{2(\beta-1)}{\beta}}>
$$




$$
>k \frac{\beta}{2}(\sqrt{2 k})^{-\frac{2(\beta-1)}{\beta}}\left|p_{2}\right|^{\frac{2(\beta-1)}{\beta}}
$$

Como no raciocínio anterior veja que $p_{2}>0$ e $\dot{p_{2}}<0$, portanto podemos escrever que

$$
\begin{gathered}
\frac{-\dot{p_{2}}}{p_{2} \frac{2(\beta-1)}{\beta}}>k \frac{\beta}{2}(\sqrt{2 k})^{-\frac{2(\beta-1)}{\beta}} \Longrightarrow\left(\frac{\beta}{\beta-2}\right) p_{2}^{\frac{-\beta+2}{\beta}}>k\left(\frac{\beta}{2}\right)(\sqrt{2 k})^{-\frac{2(\beta-1)}{\beta}} t+\overline{c_{3}} \\
\Longrightarrow 0<p_{2}<\left(k\left(\frac{\beta-2}{2}\right)(\sqrt{2 k})^{-\frac{2(\beta-1)}{\beta}} t+\tilde{c_{3}}\right)^{\frac{\beta}{-\beta+2}}
\end{gathered}
$$

Precisamos agora estimar $p_{1}$. Primeiro, procedemos como acima: pelo lema 6.2 temos $p_{1}>\left(-q_{1}\right)^{\frac{\alpha+1}{2}}$ e de $\dot{q}_{1}=\left(1+q_{1}\right) p_{1}$ temos que $\dot{q}_{1}>(1+$ $\left.q_{1}\right)\left(-q_{1}\right)^{\frac{\alpha+1}{2}}$. Lembrando que supomos inicialmente $\left|q_{1}(t)\right|<\frac{1}{2}$ para $t>0$ e como $q_{1}(t)<0$ para $t>0$ temos que

$$
\dot{q}_{1}>\frac{1}{2}\left(-q_{1}\right)^{\frac{(\alpha+1)}{2}} \Longrightarrow \frac{2}{(\alpha-1)}\left(-q_{1}\right)^{\frac{-(\alpha-1)}{2}}>\frac{1}{2} t+\overline{c_{0}}
$$

desta forma, como $\alpha>1$, note que $\left(\frac{\alpha-1}{2}\right)>0$ mas o expoente $-(\alpha-1)$ inverte a desigualdade. Assim

$$
0<\left(-q_{1}\right)<\left(\left(\frac{\alpha-1}{4}\right) t+\tilde{c_{0}}\right)^{\frac{-2}{\alpha-1}}
$$

Olhando para o nível de energia zero (que, como já vimos, é onde uma solução assintótica deve estar) e usando o lema 6.3 , veja que $H_{2}-U<0$. Logo,

$$
\begin{gathered}
\frac{\left(1+q_{1}\right)\left(p_{1}^{2}+p_{2}^{2}\right)}{2}+\frac{q_{1}\left|q_{1}\right|^{\alpha}-k\left|q_{2}\right|^{\beta}}{2}+\frac{k\left|q_{2}\right|^{\beta}-p_{2}^{2}}{2}<0 \Longrightarrow \\
\Longrightarrow\left(1+q_{1}\right) \frac{p_{1}^{2}}{2}+q_{1} \frac{p_{2}^{2}}{2}+\frac{q_{1}\left|q_{1}\right|^{\alpha}}{2}<0
\end{gathered}
$$

se notarmos que, como $-\frac{1}{2}<q_{1}(t)<0$ para todo $t>0$,

$$
\frac{p_{1}^{2}}{4}<-q_{1} \frac{p_{2}^{2}}{2}-\frac{q_{1}\left|q_{1}\right|^{\alpha}}{2}=-q_{1} \frac{p_{2}^{2}}{2}+\frac{\left|q_{1}\right|^{\alpha+1}}{2}
$$

O que implica, usando as estimativas obtidas acima, que

$$
\begin{array}{r}
\frac{p_{1}^{2}}{2}<\left(\left(\frac{\alpha-1}{4}\right) t+\tilde{c_{0}}\right)^{\frac{-2}{\alpha-1}}\left(k\left(\frac{\beta-2}{2}\right)(\sqrt{2 k})^{-\frac{2(\beta-1)}{\beta}} t+\tilde{c_{3}}\right)^{\frac{2 \beta}{-\beta+2}}+\ldots \\
\ldots+\left(\left(\frac{\alpha-1}{4}\right) t+\tilde{c_{0}}\right)^{\frac{-2(\alpha+1)}{\alpha-1}}
\end{array}
$$


Agora note que, como $k>0$, podemos isolar esse termo obtendo

$$
\left(k\left(\frac{\beta-2}{2}\right)(\sqrt{2 k})^{-\frac{2(\beta-1)}{\beta}} t+\tilde{c_{3}}\right)^{\frac{2 \beta}{-\beta+2}}=\left(k^{\frac{1}{\beta}}\right)^{\frac{2 \beta}{-\beta+2}}\left((2)^{\frac{-\beta+1}{\beta}}\left(\frac{\beta-2}{2}\right) t+\widehat{c_{3}}\right)^{\frac{2 \beta}{-\beta+2}}
$$

Como $\beta>2$ temos que $\frac{-\beta+1}{\beta}=-1+\frac{1}{\beta}>-1$ e assim $(2)^{\frac{-\beta+1}{\beta}}>\frac{1}{2}$. Além disso supomos que $\frac{-1}{\alpha-1}<\frac{2}{-\beta+2}$, logo $\frac{\beta-2}{2}>\alpha-1>\frac{\alpha-1}{2}$. Veja portanto que $2^{\frac{-\beta+1}{\beta}}\left(\frac{\beta-2}{2}\right)>\frac{1}{2} \frac{\alpha-1}{2}=\frac{\alpha-1}{4}$, assim, pelo lema 4.4.2, sabemos também que existe $t_{1}$ suficientemente grande de forma que podemos majorar, para todo $t>\max \left\{0, t_{1}\right\}$, olhando apenas para o menor fator que multiplica $t$.

$$
\left((2)^{\frac{-\beta+1}{\beta}}\left(\frac{\beta-2}{2}\right) t+\widehat{c_{3}}\right)^{\frac{2 \beta}{-\beta+2}}<\left(\left(\frac{\alpha-1}{4}\right) t+\tilde{c_{0}}\right)^{\frac{2 \beta}{-\beta+2}}
$$

e obtemos desta forma

$$
\begin{gathered}
\frac{p_{1}^{2}}{2}<\left(k^{\frac{1}{\beta}}\right)^{\frac{2 \beta}{-\beta+2}}\left(\left(\frac{\alpha-1}{4}\right) t+\tilde{c_{0}}\right)^{\frac{-2}{\alpha-1}}\left((2)^{\frac{-\beta+1}{\beta}}\left(\frac{\beta-2}{2}\right) t+\widehat{c_{3}}\right)^{\frac{2 \beta}{-\beta+2}}+\ldots \\
\ldots+\left(\left(\frac{\alpha-1}{4}\right) t+\tilde{c_{0}}\right)^{\frac{-2(\alpha+1)}{\alpha-1}}< \\
<\left(k^{\frac{1}{\beta}}\right)^{\frac{2 \beta}{-\beta+2}}\left(\left(\frac{\alpha-1}{4}\right) t+\tilde{c_{0}}\right)^{\left(\frac{-2}{\alpha-1}+\frac{2 \beta}{-\beta+2}\right)}+\left(\left(\frac{\alpha-1}{4}\right) t+\tilde{c_{0}}\right)^{\frac{-2(\alpha+1)}{\alpha-1}}
\end{gathered}
$$

Usando agora o lema 4.4.1 teremos que

$$
p_{1}^{2}<2\left(1+\left(k^{\frac{1}{\beta}}\right)^{\frac{2 \beta}{-\beta+2}}\right)\left(\left(\frac{\alpha-1}{4}\right) t+\tilde{c_{0}}\right)^{\left(\frac{-2}{\alpha-1}+\frac{2 \beta}{-\beta+2}\right)}
$$

se

$\frac{-2}{\alpha-1}+\frac{2 \beta}{-\beta+2} \geq \frac{-2(\alpha+1)}{\alpha-1} \Longleftrightarrow \frac{2 \beta}{-\beta+2} \geq \frac{-2(\alpha+1)}{\alpha-1}-\frac{-2}{\alpha-1}=\frac{-2 \alpha}{\alpha-1}$

e tomarmos $t_{2}$ suficientemente grande (pois novamente estamos majorando pelo maior expoente). Note que a condição acima $\frac{\beta}{-\beta+2} \geq \frac{-\alpha}{\alpha-1}$ é a mesma pedida inicialmente. Desta forma obtemos, finalmente, que

$$
p_{1}<\sqrt{2\left(1+k^{\frac{2}{-\beta+2}}\right)}\left(\left(\frac{\alpha-1}{4}\right) t+\tilde{c_{0}}\right)^{\left(\frac{-1}{\alpha-1}+\frac{\beta}{-\beta+2}\right)}
$$

Agora estamos em condições de completar a demonstração. Defina para $t>\max \left\{1, t_{1}, t_{2}\right\}$

$$
f(t)=\int_{t}^{\infty} \frac{p_{2}^{2}(s)}{2} d s
$$


Note também que $\dot{p_{1}}<-\frac{p_{2}^{2}}{2}$. Assim,

$$
\lim _{s \rightarrow \infty} p_{1}(s)=p_{1}(t)+\int_{t}^{\infty} \dot{p_{1}}(w) d w<p_{1}(t)-f(t)
$$

Como

$$
\begin{gathered}
f(t)=\int_{t}^{\infty} \frac{p_{2}^{2}(s)}{2}>\frac{1}{2} \int_{t}^{\infty}\left(k^{\frac{1}{\beta}}\left(\frac{\beta-2}{2}\right) s+\tilde{c_{3}}\right)^{\frac{2 \beta}{-\beta+2}}= \\
=\left.\left(\frac{-1}{k^{\frac{1}{\beta}}(\beta+2)}\left(k^{\frac{1}{\beta}}\left(\frac{\beta-2}{2}\right) s+\tilde{c_{3}}\right)^{\frac{\beta+2}{-\beta+2}}\right)\right|_{t} ^{\infty} \\
=\frac{1}{k^{\frac{1}{\beta}}(\beta+2)}\left(k^{\frac{1}{\beta}}\left(\frac{\beta-2}{2}\right) t+\tilde{c_{3}}\right)^{\frac{\beta+2}{-\beta+2}}
\end{gathered}
$$

temos que

$$
\lim _{s \rightarrow \infty} p_{1}(s)<\sqrt{2\left(1+k^{\frac{2}{-\beta+2}}\right)}\left(\left(\frac{\alpha-1}{4}\right) t+\tilde{c_{0}}\right)^{\left(\frac{-1}{\alpha-1}+\frac{\beta}{-\beta+2}\right)}+\ldots
$$

$$
\ldots-\frac{1}{k^{\frac{1}{\beta}}(\beta+2)}\left(k^{\frac{1}{\beta}}\left(\frac{\beta-2}{2}\right) t+\tilde{c_{3}}\right)^{\frac{\beta+2}{-\beta+2}}
$$

Será menor que zero sempre que $\frac{-1}{\alpha-1}+\frac{\beta}{-\beta+2}<\frac{\beta+2}{-\beta+2}$ ou seja

$$
\frac{-1}{\alpha-1}<\frac{\beta+2}{-\beta+2}-\frac{\beta}{-\beta+2}=\frac{2}{-\beta+2}
$$

pois podemos majorar, para algum $t_{3}$ suficientemente grande, passando os termos externos para dentro de seus respectivos parêntesis e usando o lema 4.4.1 para obter que, se $t>\max \left\{1, t_{1}, t_{2}, t_{3}\right\}$,

$$
\begin{gathered}
\lim _{s \rightarrow \infty} p_{1}(s)<\frac{1}{k^{\frac{1}{\beta}}(\beta+2)}\left(k^{\frac{1}{\beta}}\left(\frac{\beta-2}{2}\right) t+\tilde{c_{3}}\right)^{\frac{\beta+2}{-\beta+2}}+\ldots \\
\ldots-\frac{1}{k^{\frac{1}{\beta}}(\beta+2)}\left(k^{\frac{1}{\beta}}\left(\frac{\beta-2}{2}\right) t+\tilde{c_{3}}\right)^{\frac{\beta+2}{-\beta+2}}=0
\end{gathered}
$$

Em particular, podemos melhorar esse resultado usando o lema 4.4.2 para incluir o caso $\frac{-1}{\alpha-1}=\frac{2}{-\beta+2}$ se $k>0$ for escolhido de forma que, em (6.4) , o fator que multiplica $t$ no primeiro termo seja maior do que o fator que multiplica $t$ no segundo termo, ou seja, de forma que 


$$
\left(\frac{1}{k^{\frac{1}{\beta}}}\right)^{\frac{-\beta+2}{\beta+2}} k^{\frac{1}{\beta}}\left(\frac{1}{\beta+2}\right)^{\frac{-\beta+2}{\beta+2}}\left(\frac{\beta-2}{2}\right)<{\sqrt{2\left(1+k^{\frac{2}{-\beta+2}}\right)}}^{\frac{-\beta+2}{\beta+2}}\left(\frac{\alpha-1}{4}\right)
$$

Mas deixaremos essa análise para o apêndice ao final dessa dissertação, assim como fizemos anteriormente.

Portanto, começamos supondo que $\phi(t)$ satisfazia $\lim _{t \rightarrow+\infty}\|\phi(t)\|=0$, mas obtivemos que, sob as condições enunciadas, $\lim _{s \rightarrow \infty} p_{1}(s)<0$. Isso é uma clara contradição, o que conclui toda a demonstração.

Corolário 6.6. Suponha que $\phi(t)=\left(q_{1}(t), q_{2}(t), p_{1}(t), p_{2}(t)\right)$ é solução de (6.3) tal que $\lim _{t \rightarrow+\infty}\|\phi(t)\|=0$. Se $1<\alpha<\frac{\beta}{2}$ então $p_{2}(t)=q_{2}(t)=0$ para todo $t$. Se em particular $k$ for escolhido como no teorema o resultado valerá também para $\alpha=\frac{\beta}{2}$.

Demonstração. A demonstração consiste em observar que, se $\alpha<\frac{\beta}{2}$ as duas hipóteses principais ficam automaticamente verificadas.

Para isso considere a função $g(\alpha, \beta)=\frac{-\alpha}{\alpha-1}-\frac{\beta}{-\beta+2}$. Queremos provar que $g(\alpha, \beta)<0$, o que fará com que uma das hipóteses do teorema esteja automaticamente verificada. Os zeros dessa função satisfazem

$$
\frac{-\alpha}{\alpha-1}=\frac{\beta}{-\beta+2} \Leftrightarrow \frac{\alpha-1}{-\alpha}=\frac{-\beta+2}{\beta} \Leftrightarrow-1+\frac{1}{\alpha}=-1+\frac{2}{\beta} \Leftrightarrow \alpha=\frac{\beta}{2}
$$

pois $\alpha, \beta>0$. Como o sinal que $g(\alpha, \beta)$ não muda para $\beta>2 \alpha$ calculamos, por exemplo,

$$
\begin{aligned}
g(\alpha, 4 \alpha) & =\frac{-\alpha}{\alpha-1}-\frac{4 \alpha}{-4 \alpha+2}=\frac{-\alpha}{\alpha-1}+\frac{2 \alpha}{2 \alpha-1}= \\
& =\frac{-\alpha(2 \alpha-1)+2 \alpha(\alpha-1)}{(\alpha-1)(2 \alpha-1)}=\frac{-\alpha}{(\alpha-1)(2 \alpha-1)}<0
\end{aligned}
$$

Finalmente, note que $\alpha \leq \frac{\beta}{2}$ equivale a hipótese $\frac{-1}{\alpha-1} \leq \frac{2}{-\beta+2}$, o que completa a demonstração.

Corolário 6.7. Suponha que $\phi(t)=\left(q_{1}(t), q_{2}(t), p_{1}(t), p_{2}(t)\right)$ é solução de (6.3) tal que $\lim _{t \rightarrow+\infty}\|\phi(t)\|=0$. Se $1<\alpha \leq \frac{2 \beta-2}{\beta}$ então $p_{2}(t)=q_{2}(t)=0$ para todo $t$.

Demonstração. A demonstração consiste em observar que, como $\beta>2$ (pois $1<\frac{2 \beta-2}{\beta}$ ) as duas hipóteses principais ficam automaticamente verificadas. De fato $\alpha<\frac{2 \beta-2}{\beta}$ implica que $\alpha-1<\frac{\beta-2}{\beta}$ ou seja que $\frac{-1}{\alpha-1}<\frac{\beta}{-\beta+2}$. Como $\frac{\beta}{-\beta+2}<\frac{2}{-\beta+2}($ pois $\beta>2$ e $-\beta+2<0)$ e $\frac{-1}{\alpha-1}>\frac{-\alpha}{\alpha-1}($ pois $\alpha>1 \mathrm{e}$ $\alpha-1>0)$ então $\frac{2}{-\beta+2}>\frac{\beta}{-\beta+2}>\frac{-1}{\alpha-1}>\frac{-\alpha}{\alpha-1}$. 
Nos corolários acima note que quando $\alpha \searrow 1$ podemos tomar $\beta \searrow 2$ garantimos que a condição $\beta>2$. Assim, é suficiente para produzirmos (um contínuo de) exemplos em que as hipóteses do teorema acima são satisfeitas. Em outras palavras, dado $\beta>2$ existe $\alpha>1$ que satisfaz as condições acima e vice-versa. Esse fato é de extrema importância se notarmos que as hipóteses $\alpha>1$ e $\beta>2$ eram apenas as hipóteses de regularidade exigidas a priori. Essas condições só não são mínimas pois ainda poderíamos ter estudado o caso em que $\beta=2$, mas o deixamos de lado devido a natureza tão distinta de suas soluções assintóticas à origem.

É natural nos perguntarmos qual das estimativas produz mais informação. Em outras palavras, queremos que, dado $\beta>2, \alpha<\max \left\{\frac{\beta}{2}, 2-\frac{2}{\beta}\right\}$ (e, consequentemente, $\left.\beta>\min \left\{2 \alpha, \frac{-2}{\alpha-2}\right\}\right)$. Veja que $2-\frac{2}{\beta}-\frac{\beta}{2}=\frac{4 \beta-4-\beta^{2}}{2 \beta}<0$ (concavidade para baixo, vértice em $\beta=2$ ), portanto $2-\frac{2}{\beta}<\frac{\beta}{2}$ para $\beta>2$. Em particular, provamos que o primeiro corolário engloba o segundo (este aparece aqui apenas como uma outra forma de analisar o problema e para seu devido registro).

Escrevemos explicitamente um corolário que apenas resume o que discutimos até agora.

Corolário 6.8. Dado $\alpha>1$ a dimensão da bacia de atração da origem para as equações de $\mathrm{H}_{1}$ e $\mathrm{H}_{2}$ é diferente sempre que $\alpha \leq \frac{\beta}{2}$ (caso $\alpha \geq 2$ e a igualdade valendo apenas se $k$ for escolhido adequadamente).

Demonstração. É uma simples consequência do lema 6.1 que $B A_{1}$ é uma subvariedade de $\mathbb{R}^{4}$ de dimensão 2 (pois $q_{1}=f\left(p_{1}\right)$ e $q_{2}=g\left(p_{2}\right)$ ).

Por sua vez os corolários acima dizem que, sob as hipóteses enunciadas, uma solução para $H_{2}$ assintótica à origem deve satisfazer $p_{2}(t)=q_{2}(t)=0$ para todo $t$. Assim a dimensão da bacia de atração da origem para $H_{2}$ é 1 .

Portanto as dimensões das bacias de atração são diferentes sempre que respeitadas as condições do teorema 6.5.

Desta forma obtivemos o resultado principal deste trabalho: um critério explícito que mostra quando uma família de hamiltonianos dados por uma família específica de energias potenciais é perturbado (no sentido da bacia de atração) pela mudança da energia cinética euclidiana $T_{1}$ para a energia cinética $T_{2}=\left(1+q_{1}\right) T_{1}$, o que conclui este capítulo. 


\section{Capítulo 7}

\section{Conclusões}

Nosso objetivo neste trabalho foi estudar a segunda parte do artigo de Garcia \& Tal [5] sobre a influência da energia cinética na análise da estabilidade em sistemas hamiltonianos. Apesar de não tratarmos diretamente da estabilidade segundo Liapunov, nossa análise fornece indício de alguma influência da energia cinética, já que, mesmo para casos analíticos, conseguimos obter uma família de potenciais cuja bacia de atração é perturbada (no sentido de sua dimensão como subvariedade de $\mathbb{R}^{4}$ ) pela mudança de energia cinética que estudamos. Como discutimos anteriormente, nosso conhecimento atual sobre o assunto limita a influência da energia cinética na estabilidade segundo Liapunov. Mesmo assim, na busca de uma "inversão" para o teorema de Dirichlet-Lagrange já sabemos, devido aos resultados de Bertotti \& Bolotin [3] e Garcia \& Tal [5] , que a energia cinética deve, de alguma forma, ser incluída.

Nosso método foi manipular os expoentes dos termos que aparecem na energia potencial originalmente proposta, generalizar os lemas e utilizar toda a força das desigualdades propostas. O primeiro passo foi reduzir um dos expoentes, já que este havia sido escolhido de forma mais solta, apenas de forma que garantisse as desigualdades fornecidas pelos autores. Em particular descobrimos que o menor expoente possível no artigo original era $\beta=6$, que foi a questão que deu origem a este trabalho. O segundo passo foi determinar um critério algébrico entre os dois expoentes que garatisse o resultado desejado.

A partir disso obtivemos o que é o resultado principal dessa dissertação, que é a demonstração de que dado um parâmetro real é possível gerar um contínuo de exemplos em que vale o resultado original. Mais ainda, mostramos que, a menos do caso $\beta=2$ que não foi tratado, a escolha desse parâmetro pode ser feita dentro de um conjunto que corresponde apenas a hipóteses mínimas de regularidade.

Nosso resultado abre algumas questões

1. Vale o mesmo resultado, por exemplo, para o potencial $H(q, p)=$ 
$\frac{q_{1}^{3}-q_{2}^{4}}{2} ?$ Em geral, o que acontece se os expoentes não obedecem ao critério obtido?

2. Vale o resultado se a série de Taylor (de ordem apropriada) da energia potencial coincide com uma função de nossa família que respeita o critério obtido?

3. Como completar essa demonstração acrescentando o caso $\beta=2$ ? (Isso, é claro, se o resultado for mesmo válido!)

4. Existem outras energias cinéticas que proporcionam o mesmo resultado?

Sobre o primeiro item ainda sabemos pouco. Principalmente porque o raciocínio que usamos aqui usa estimativas razoavelmente precisas, que de fato tentamos levar ao limite com esse trabalho.

A resposta para o segundo item, aparente, pode ser obtida com uma análise mais profunda deste trabalho. Não é difícil validar automaticamente alguns pontos da demonstração principal, mas outros pontos não parecem tão simples de serem validados. A análise formal dessa questão é um problema que deixo para o futuro.

O terceiro item é realmente intrigante. É razoável crer que ou o resultado seguirá de algumas desigualdade simples relacionando as funções exponenciais e as funções racionais ou exigirá uma abordagem nova consideravelmente diferente. Sem esquecer que é perfeitamente possível que um resultado análogo não valha.

Finalmente, se o leitor permitir alguma ousadia, eu diria que para o quarto item a resposta é sim. Na verdade é minha otimista opinião que a energia cinética ainda possui surpresas para revelar, dentro do espectro de surpresas que podemos esperar. É meu desejo continuar a desvendar esse mistério nos anos que virão e, quem sabe, o caro leitor me acompanhará na conclusão dessa próxima etapa. 


\section{Capítulo 8}

\section{Apêndice}

Neste apêndice explicitamos os cálculos que deixamos de lado nos capítulos 5 e 6. Apesar deles permitem uma análise mais profunda dos teoremas principais desta dissertação seu cálculo exato durante uma demonstração longa e delicada não seria interessante, ainda mais porque se tratam apenas de cálculos algébricos que não acrescentam idéias importantes.

\subsection{Apêndice ao capítulo 5}

Como já deixamos indicado na penúltima página do capítulo 5, queremos ver se existe $k>0$ de forma que

$$
\left(\frac{1}{6 k^{\frac{1}{4}}}\right)^{-\frac{1}{3}} k^{\frac{1}{4}}<\frac{1}{4}{\sqrt{2\left(1+k^{-1}\right)}}^{-\frac{1}{3}}
$$

Para isso analisamos a função $f(k)=\left(\frac{1}{6 k^{\frac{1}{4}}}\right)^{-\frac{1}{3}} k^{\frac{1}{4}}-\frac{1}{4} \sqrt{2\left(1+k^{-1}\right)}-\frac{1}{3}$. Seus zeros satisfazem

$$
4\left(\frac{1}{6 k^{\frac{1}{4}}}\right)^{-\frac{1}{3}} k^{\frac{1}{4}}={\sqrt{2\left(1+k^{-1}\right)}}^{-\frac{1}{3}} \Longleftrightarrow 4\left(\frac{1}{6 k}\right)^{-\frac{1}{3}}=\left(2+\frac{2}{k}\right)^{-\frac{1}{6}}
$$

Que por sua vez equivale a

$$
4^{-6}\left(\frac{1}{6 k}\right)^{2}=\left(2+\frac{2}{k}\right) \Longleftrightarrow \frac{1}{4^{6}} \frac{1}{6^{2}} k^{-2}-2 k^{-1}-2=0
$$

Fazendo a substituição $x=k^{-1}$ obtemos $\Delta=4-4\left(\frac{1}{4^{6} 6^{2}}\right)(-2)=4+\frac{2}{4^{5} 6^{2}}$. Como $\sqrt{\Delta}>\sqrt{4}=2$ temos apenas uma solução positiva

$$
x=\frac{2+\sqrt{\Delta}}{\frac{2}{4^{6} 6^{2}}}=\frac{4^{6} 6^{2}}{2}\left(2+\sqrt{4+\frac{2}{4^{5} 6^{2}}}\right)>4^{6} 6^{2} 2=2^{15} 3^{2}
$$


Para determinar o sinal de $f(k)$ em cada um dos intervalos determinados por $\frac{1}{x} \approx 3.3 * 10^{-6}$ calculamos, por exemplo

$$
\begin{gathered}
f\left(10^{-6}\right)=\left(\frac{10^{6}}{6}\right)^{-\frac{1}{3}}-\frac{1}{4}\left(2+\frac{2}{10^{-6}}\right)^{-\frac{1}{6}}<0.02-0.02=0 \\
f(1)=\left(\frac{1}{6}\right)^{-\frac{1}{3}}-\frac{1}{4} 4^{-\frac{1}{6}}>1.8-0.2>0
\end{gathered}
$$

Desta forma $f(k)<0$ para $0<k<\frac{1}{2^{15} 3^{2}}$

\subsection{Apêndice ao capítulo 6}

A segunda desigualdade que deixamos pendente é análoga a do capítulo 5 . Queremos determinar $k>0$ de forma que

$$
\left(\frac{1}{k^{\frac{1}{\beta}}}\right)^{\frac{-\beta+2}{\beta+2}} k^{\frac{1}{\beta}}\left(\frac{1}{\beta+2}\right)^{\frac{-\beta+2}{\beta+2}}\left(\frac{\beta-2}{2}\right)<{\sqrt{2\left(1+k^{\frac{2}{\beta+2}}\right)}}^{\frac{-\beta+2}{\beta+2}}\left(\frac{\alpha-1}{4}\right)
$$

Veja que

$$
\left(\frac{1}{k^{\frac{1}{\beta}}}\right)^{\frac{-\beta+2}{\beta+2}} k^{\frac{1}{\beta}}=\left(k^{\frac{-1}{\beta}} k^{\frac{1}{\beta} \frac{\beta+2}{-\beta+2}}\right)^{\frac{-\beta+2}{\beta+2}}=\left(k^{\frac{2}{-\beta+2}}\right)^{\frac{-\beta+2}{\beta+2}}=k^{\frac{2}{\beta+2}}
$$

Procuramos então os zeros da função

$$
f(k)=k^{\frac{2}{\beta+2}}\left(\frac{1}{\beta+2}\right)^{\frac{-\beta+2}{\beta+2}}\left(\frac{\beta-2}{2}\right)-\left(2+2 k^{\frac{2}{-\beta+2}}\right)^{\frac{-\beta+2}{2(\beta+2)}}\left(\frac{\alpha-1}{4}\right)
$$

observando que

$$
k^{\frac{4}{-\beta+2}}\left(\frac{1}{\beta+2}\right)^{2}\left(\frac{\beta-2}{2}\right)^{\frac{2(\beta+2)}{-\beta+2}}=\left(2+2 k^{\frac{2}{-\beta+2}}\right)\left(\frac{\alpha-1}{4}\right)^{\frac{2(\beta+2)}{-\beta+2}}
$$

e fazendo a substituição $x=k^{\frac{2}{-\beta+2}}$ obtemos

$$
\left(\frac{1}{\beta+2}\right)^{2}\left(\frac{\beta-2}{2}\right)^{\frac{2(\beta+2)}{-\beta+2}} x^{2}-2\left(\frac{\alpha-1}{4}\right)^{\frac{2(\beta+2)}{-\beta+2}} x-2\left(\frac{\alpha-1}{4}\right)^{\frac{2(\beta+2)}{-\beta+2}}=0
$$

ou ainda

$$
\left(\frac{1}{\beta+2}\right)^{2}\left(\frac{2(\beta-2)}{\alpha-1}\right)^{\frac{2(\beta+2)}{-\beta+2}} x^{2}-2 x-2=0
$$


desta forma $\Delta=4-4(-2)\left(\frac{1}{\beta+2}\right)^{2}\left(\frac{2(\beta-2)}{\alpha-1}\right)^{\frac{2(\beta+2)}{-\beta+2}}$. Como $\sqrt{\Delta}>\sqrt{4}=2$ temos apenas uma solução positiva

$$
x=(\beta+2)^{2}\left(\frac{2(\beta-2)}{\alpha-1}\right)^{\frac{-2(\beta+2)}{-\beta+2}}\left(1+\sqrt{1+2\left(\frac{1}{\beta+2}\right)^{2}\left(\frac{2(\beta-2)}{\alpha-1}\right)^{\frac{2(\beta+2)}{-\beta+2}}}\right)
$$

Falta agora calcular o sinal de $f(s)$ nos intervalos $0<s<x^{\frac{-\beta+2}{2}}$ e $s>$ $x^{\frac{-\beta+2}{2}}$. Queremos mostrar que, para quaisquer $\alpha, \beta$ dados, temos $f(k)<0$ para todo $k$ suficientemente pequeno. Para isso supomos que para todo $\varepsilon>0$ exista $0<k<\varepsilon$ tal que

$$
C_{1} k^{\frac{2}{\beta+2}} \geq C_{2}\left(2+2 k^{\frac{2}{-\beta+2}}\right)^{\frac{-\beta+2}{2(\beta+2)}}
$$

onde $C_{1}=\left(\frac{1}{\beta+2}\right)^{\frac{-\beta+2}{\beta+2}}\left(\frac{\beta-2}{2}\right)$ e $C_{2}=\left(\frac{\alpha-1}{4}\right)$. Assim, teríamos, como $\frac{\beta+2}{2}>0$, que

$$
C_{3} k \geq C_{4}\left(1+k^{\frac{2}{-\beta+2}}\right)^{\frac{-\beta+2}{4}}
$$

onde $C_{3}, C_{4}>0$ são as constantes adequadas. Contudo, isso não pode occorer, pois para $k$ suficientemente pequeno temos que $\left(1+k^{\frac{2}{-\beta+2}}\right)^{\frac{-\beta+2}{4}} \geq$ $\left(2 k^{\frac{2}{-\beta+2}}\right)^{\frac{-\beta+2}{4}}, \log \mathrm{O}$

$$
C_{3} k \geq C_{5} k^{\frac{1}{2}}
$$

para algum $0<k<\varepsilon$, o que é um absurdo. Desta forma, como $f$ só pode mudar de sinal no ponto calculado acima, temos que $f(k)<0$ apenas para

$$
k<\left(( \beta + 2 ) ^ { 2 } ( \frac { 2 ( \beta - 2 ) } { \alpha - 1 } ) ^ { \frac { 2 ( \beta + 2 ) } { - \beta + 2 } } \left(1+\sqrt{\left.\left.1+2\left(\frac{1}{\beta+2}\right)^{2}\left(\frac{2(\beta-2)}{\alpha-1}\right)^{\frac{2(\beta+2)}{-\beta+2}}\right)\right)^{\frac{-\beta+2}{2}}}\right.\right.
$$

conforme utilizamos durante o texto. 


\section{Referências Bibliográficas}

[1] Vladimir I. Arnold, Ordinary differential equations, The MIT Press, 1978.

[2] Ivan de Q. Barros and Manuel V. P. Garcia, Mecânica analítica clássica, Edgar Blücher LTDA, São Paulo, 1995.

[3] Maria Letizia Bertotti and Sergey V. Bolotin, On the influence of the kinetic energy on the stability of equilibria of natural Lagrangian systems, Arch. Ration. Mech. Anal. 152 (2000), no. 1, 65-79. MR MR1760665 (2001b:37080)

[4] Claus I. Doering and Artur O. Lopes, Equações diferenciais ordinárias, Coleção matemática universitária, IMPA, Rio de Janeiro, 2005.

[5] M. V. P. Garcia and F. A. Tal, The influence of the kinetic energy in equilibrium of Hamiltonian systems, J. Differential Equations 213 (2005), no. 2, 410-417. MR MR2142373 (2006a:37051)

[6] Manuel V. P. Garcia and Fábio A. Tal, Stability of equilibrium of conservative systems with two degrees of freedom, J. Differential Equations 194 (2003), no. 2, 364-381. MR MR2006217 (2004g:70040)

[7] P. Hagedorn, Die umkehrung der stabilitätssätze von lagrange-dirichlet und routh, Arch. Rational Mech. Anal. 42 (1971), 281-316.

[8] Peter Hagedorn and Jean Mawhin, A simple variational approach to a converse of the Lagrange-Dirichlet theorem, Arch. Rational Mech. Anal. 120 (1992), no. 4, 327-335. MR MR1185564 (94a:34070)

[9] Artur O. Lopes, Introdução à mecânica clássica, EDUSP, São Paulo, 2006.

[10] C. Maffei, V. Moauro, and P. Negrini, On the inversion of the LagrangeDirichlet theorem in a case of nonhomogeneous potential, Differential Integral Equations 4 (1991), no. 4, 767-782. MR MR1108059 (92c:70024) 
[11] Vinicio Moauro and Piero Negrini, On the inversion of LagrangeDirichlet theorem, Differential Integral Equations 2 (1989), no. 4, 471478. MR MR996754 (90g:70017)

[12] Angelo Barone Netto, Jet-detectable extrema, Proceedings of the AMS 92 (1984), no. 4, 604-608.

[13] P. Painlevé, Sur la stabilité de l'équilibre, Académie des Sciences (1904), $1555-1557$.

[14] V. P. Palamodov, Stability of motion and algebraic geometry, Dynamical systems in classical mechanics, Amer. Math. Soc. Transl. Ser. 2, vol. 168, Amer. Math. Soc., Providence, RI, 1995, pp. 5-20. MR MR1351031 (97b:34055)

[15] Nicolas Rouche, P. Habets, and M. Laloy, Stability theory by Liapunov's direct method, Springer-Verlag, New York, 1977, Applied Mathematical Sciences, Vol. 22. MR MR0450715 (56 \#9008)

[16] Steven D. Taliaferro, An inversion of the Lagrange-Dirichlet stability theorem, Arch. Rational Mech. Anal. 73 (1980), no. 2, 183-190. MR MR556561 (81c:70012) 